\title{
Mappings and homological properties in the Conley index theory
}

\author{
CHRISTOPHER K. McCORD
}

Department of Mathematics, University of Cincinnati, Cincinnati, Ohio 45221, USA

\begin{abstract}
The role in the Conley index of mappings between flows is considered. A class of maps is introduced which induce maps on the index level. With the addition of such maps to the theory, the homology Conley index becomes a homology theory. Using this structure, an analogue of the Lefschetz theorem is proved for the Conley index. This gives a new condition for detecting fixed points of flows, extending the classical Euler characteristic condition.
\end{abstract}

\section{Introduction}

The techniques of algebraic topology have long been useful in the study of dynamical systems. Two classical results are the Lefschetz-Hopf fixed-point theorem and the Morse inequalities. The Lefschetz theorem has as a corollary a condition for the existence of fixed points of a flow on a compact polyhedron $X$. Namely, if the Euler characteristic $\chi(X) \neq 0$, then every flow on $X$ has a fixed point. The Morse inequalities (among other things) strengthen this result for gradient-like flows on $X$, relating $\chi(X)$ to the number of fixed points.

The Conley index theory generalizes Morse theory in two ways: it generalizes hyperbolic critical points to isolated invariant sets; and it greatly weakens the conditions on the flow needed for the index to be defined. The Conley index is the homotopy type of a pointed space $N / N_{0}$, where $\left(N, N_{0}\right)$ is an index pair for $S$ in $X$ : a compact pair chosen to capture the behaviour of the flow about $S$ in $X$. The homology of $N / N_{0}$ then defines the homology Conley index, denoted here by $\mathrm{CH}_{*}(\mathrm{X} ; \mathrm{S})$. With the Poincare polynomials of these homology groups replacing the Morse indices of hyperbolic critical points, the Morse inequality remains valid [2]. This and the other uses of the homology index ([1], [4]-[8]) have made it a powerful topological tool for dynamical systems.

This work seeks to further strengthen the Conley index theory by introducing to it mappings between flows. A class of maps, called flow maps, is defined and shown to induce maps between Conley indices and between homology Conley indices. This enables invariant sets in different flows to be compared in the Conley index theory. In particular, with the addition of flow maps to the theory, we can define a category of isolated invariant sets on which the Conley index is a functor and the homology Conley index is a homology theory. This greatly increases the range of homological methods available to the index theory, and generates analogues of many of the standard theorems of homology.

One of these is a generalization of the Lefschetz fixed-point theorem. In some cases, a flow map which is also a self-map on an isolated invariant set $S$ induces a 
self-map on the homology Conley index of $S$. Using this homology in place of the singular homology of $S$, and ANR hypotheses on index pairs replacing the ANR hypothesis on $S$, we can define a generalization of the Lefschetz number, called the Conley-Lefschetz number. The generalization of the Lefschetz theorem (theorem 5.7) is that if the Conley-Lefschetz number of a map is non-zero, then a certain family of maps homotopic to it all have fixed points in $S$. In particular, the Conley-Lefschetz number of the identity (the Conley-Euler number of $S$ ) detects fixed points of the flow on $S$.

In $\S 1$ the definitions and notation of the Conley index are reviewed. In $\S 2$ we introduce mappings between flows and consider how the objects in the index theory transform under them. These maps are used in $\S 3$ to define a cateogry $\mathscr{I S}$ of isolated sets. The Conley index is shown to be a functor, and the homology Conley index a homology functor, on this category. In $\S \S 4$ and 5 we record some of the results made available by this formalism. Specifically, analogues of the Mayer-Vietoris and Künneth theorems are proved in $\S 4$, and an analogue of the Lefschetz theorem is proved in $\S 5$. This is used to show that, under mild hypotheses, if the Euler characteristic of $\mathrm{CH}_{*}(X ; S)$ is non-zero, then $S$ contains fixed points of the flow. $A$ relative version of the theorem and continuation properties of the theorem are proved. The interaction between mappings and Morse decompositions is not discussed here, but will be examined in [9].

\section{Definitions and notation}

We will use the formulation and notation of the Conley index found in Salamon ([12]). We will work only with locally compact metric flows: a locally compact metric space $X$ with a continuous action $\varphi: X \times \mathbb{R} \rightarrow X$. We will generally not display the action, writing $x \cdot t$ for $\varphi_{t}(x)$.

Definition 1.1. If $X$ is a flow, $U \subseteq X$, the maximal invariant set in $U$ is $I(U)=$ $\{x \in X \mid x \cdot \mathbb{R} \subseteq U\}$. A set $S \subseteq X$ is an isolated invariant set if there exists a compact neighbourhood $N$ of $S$ in $X$ such that $S$ is the maximal invariant set of $N$. $N$ is then an isolating neighbourhood for $S$.

For closed $U, I(U)$ is closed. In particular, the definition requires an isolated invariant set to be compact.

Definition 1.2. Let $S \subseteq X$ be an isolated invariant set. An index pair for $S$ in $X$ is a compact pair $\left(N_{1}, N_{0}\right)$ such that

(i) $\operatorname{cl}_{X}\left(N_{1} \backslash N_{0}\right)$ is an isolating neighbourhood for $S$ in $X$;

(ii) if $x \in N_{0}, t>0$ with $x \cdot[0, t] \subseteq N_{1}$, then $x \cdot[0, t] \subseteq N_{0}$;

(iii) if $x \in N_{1}$ so that $x \cdot \mathbb{R}^{+} \not \subset N_{1}$, then there exists a $t \geq 0$ such that $x \cdot[0, t] \subseteq N_{1}$ and $x \cdot t \in N_{0}$.

Property (ii) is referred to as positive invariance of $N_{0}$ in $N_{1}$; property (iii) is referred to by saying that $N_{0}$ is an exit set for $N_{1}$.

THEOREM 1.3 ([12], theorem 4.3). If $N$ is an isolating neighbourhood for $S$ in $X$, then there exists an index pair $\left(N_{1}, N_{0}\right)$ for $S$ in $X$ with $N_{1} \subseteq N$ and both $N_{1}$ and $N_{0}$ positively invariant in $N$. 
It is useful in topology to be able to work with neighbourhood deformation retract (NDR) pairs ([3], [13]). Towards this end, Salamon introduces in [12] the idea of a regular index pair, defined as follows. For any index pair $\left(N_{1}, N_{0}\right)$, define the 'exit-time' map $\tau_{+}: N_{1} \rightarrow[0, \infty]$ by

$$
\tau_{+}(x)= \begin{cases}\sup \left\{t \geq 0 \mid x \cdot[0, t] \subseteq N_{1} \backslash N_{0}\right\} & \text { if } x \in N_{1} \backslash N_{0}, \\ 0 & \text { if } x \in N_{0}\end{cases}
$$

$\left(N_{1}, N_{0}\right)$ is said to be a regular index pair if $\tau_{+}$is continuous. A regular index pair is then an NDR pair, with the neighbourhood of $N_{0}$ retracting to $N_{0}$ along flow lines. Salamon proves the following:

LeMma $1.4\left([12]\right.$, lemma 5.2). If $\left(N_{1}, N_{0}\right)$ is an index pair such that, for every $x \in N_{0}$ and every $t \geq 0, x \cdot[0, t] \not \subset \mathrm{cl}_{X}\left(N_{1} \backslash N_{0}\right)$, then $\left(N_{1}, N_{0}\right)$ is a regular index pair.

Lемма 1.5 ([12], lemma 5.3). If $\left(N_{1}, N_{0}\right)$ is an index pair for $S$ in $X$, there exists a compact $M$ such that $N_{0} \subseteq M \subseteq N_{1}$ and $\left(N_{1}, M\right)$ is an index pair which satisfies the hypothesis of 1.4 .

If $\left(N_{1}, N_{0}\right)$ is an index pair satisfying the hypothesis of 1.4 , then $\left(\tilde{N}_{1}, \tilde{N}_{0}\right)=$ $\left(\mathrm{cl}_{x}\left(N_{1} \backslash N_{0}\right), N_{0} \cap \mathrm{cl}_{x}\left(N_{1} \backslash N_{0}\right)\right)$ is also an index pair, with $\tau_{+}$continuous and $\tau_{+}(x)=\sup \left\{t \geq 0 \mid x \cdot[0, t] \subseteq \tilde{N}_{1}\right\}$. As $X$ is a two-sided flow, we can make a similar construction for $S$ as an isolated invariant set in the reverse flow (the 'exit-time' map is now an 'entrance-time' map and is denoted $\tau_{-}$). In fact, we can construct such index pairs for the forward and reverse flows simultaneously. Namely:

THEOREM 1.6. If $S$ is an isolated invariant set in a (two-sided) flow, then there exists an isolating neighbourhood $N$ and compact $N_{0}, N^{0} \subseteq \partial_{X} N$ so that

(i) $\left(N, N_{0}\right)$ is an index pair for $S$ in the forward flow with $\tau_{+}$continuous and $\tau_{+}(x)=\sup \{t \geq 0 \mid x:[0, t] \subseteq N\}$

(ii) $\left(N, N^{0}\right)$ is an index pair for $S$ in the reverse flow with $\tau_{-}$continuous and $\tau_{-}(x)=\inf \{t \leq 0 \mid x \cdot[t, 0] \subseteq N\}$.

Proof. Take an index pair $\left(\tilde{N}, \tilde{N}_{0}\right)$ satisfying (i) (which exists from the discussion above). Let $\left(\tilde{M}, \tilde{M}^{0}\right)$ be an index pair for $S$ in the reverse flow so that $\left(\tilde{M}, \tilde{M}^{0}\right)$ is negatively invariant relative to $\tilde{N}$. From Salamon's construction ([12], lemma 4.2), $\tilde{M}$ can be chosen to be a neighbourhood of $M^{+}(\tilde{N})=\left\{x \in \tilde{N} \mid x \cdot \mathbb{R}^{+} \subseteq \tilde{N}\right\}$ in $\tilde{N}$ so that $P=\left\{x \in \tilde{N} \mid \exists t \leq 0\right.$ with $x \cdot[t, 0] \subseteq \tilde{N}$ and $\left.x \cdot t \in \partial_{\tilde{N}}(\tilde{M})\right\}$ is compact. Let $M=P \cup \operatorname{cl}_{X}\left(\tilde{M} \backslash \tilde{M}^{0}\right)$.

(i) $N$ is invariant relative to $\mathrm{cl}_{X}\left(\tilde{N} \backslash \tilde{M}^{0}\right)$.

$P f$. $P$ is by construction positively invariant in $\tilde{N}$, and an orbit in $\mathrm{cl}_{X}\left(\tilde{M} \backslash \tilde{M}^{0}\right)$ can only exit $\mathrm{cl}_{X}\left(\tilde{M} \backslash \tilde{M}^{0}\right)$ if it enters $P$, so $M$ is positively invariant. Similarly, $\operatorname{cl}_{X}\left(\tilde{M} \backslash \tilde{M}^{0}\right)$ is negatively invariant in $\mathrm{cl}_{X}\left(\tilde{N} \backslash \tilde{M}^{0}\right)$ (as $\tilde{M}$ and $\tilde{M}^{0}$ are both negatively invariant in $\tilde{N})$, and orbits in $P$ can only exit $P$ in backwards time if they enter $\tilde{M}$ or $\tilde{M}^{0}$, so $M$ is negatively invariant.

(ii) $\left(M, \tilde{M}^{0}\right)$ is an index pair for $S$ in the reverse flow.

Pf. $\left(M, \tilde{M}^{0}\right)$ is formed by attaching $P$ to $\left(\tilde{M}, \tilde{M}^{0}\right) . \tilde{M} \backslash \tilde{M}^{0} \subseteq M \backslash \tilde{M} \tilde{M}^{0} \subseteq \tilde{N}$, so 
$\operatorname{cl}_{X}\left(M \backslash \tilde{M}^{0}\right)$ is an isolating neighbourhood for $S$ in $X$. From the discussion above, $\tilde{M}_{0}$ is a negatively invariant exit set for the reverse flow.

(iii) Choose an $\tilde{M}^{0} \subseteq M^{0} \subseteq M$ so that $\left(M, M^{0}\right)$ satisfies the hypothesis of 1.4 . Let $N=\mathrm{cl}_{X}\left(M \backslash M^{0}\right), N_{0}=\tilde{N}_{0} \cap N, N^{0}=M^{0} \cap N$. By construction, $\left(N, N^{0}\right)$ satisfies (ii). $\left(N, N_{0}\right)$ is the intersection of $\left(\tilde{N}, \tilde{N}_{0}\right)$ with a compact set positively invariant relative to $\tilde{N}$. Such an intersection changes none of the properties required in (i); $\left(N, N_{0}\right)$ satisfies (i).

If $S$ is isolated in $X$, the collection of index pairs $\left(N, N_{0}\right)$ which admit an $N^{0}$ so that $N, N_{0}$ and $N^{0}$ satisfy $1.6(\mathrm{i})$, (ii) will be denoted $\mathcal{N}(X ; S)$. Let $\mathscr{A} \mathcal{N}(X ; S)=$ $\left\{\left(N, N_{0}\right) \in \mathcal{N}(X ; S) \mid N / N_{0}\right.$ is an absolute neighbourhood retract (ANR; cf. [3], [13])\}. The construction of index pairs in [11] shows that $\mathscr{A N}(X ; S)$ is non-empty when $X$ is a manifold.

Index pairs are used to define the Conley index, which has the structure of a connected simple system.

Definition 1.7. A connected simple system is a collection $I_{0}$ of pointed spaces along with a collection $I_{m}$ of homotopy classes of maps between these such that

(i) $\operatorname{hom}(X, Y)=\left\{[f] \in[X, Y] \mid[f] \in I_{m}\right\}$ consists of a single element for every $X, Y \in I_{0}$;

(ii) if $X, Y, Z \in I_{0}$ and $[f] \in \operatorname{hom}(X, Y),[g] \in \operatorname{hom}(Y, Z)$, then $[g \circ f] \epsilon$ hom $(X, Z)$;

(iii) $\operatorname{hom}(X, X)=\left\{\left[\mathrm{id}_{X}\right]\right\}$ for every $X \in I_{0}$.

A morphism $\Phi:\left(I_{0}, I_{m}\right) \rightarrow\left(J_{0}, J_{m}\right)$ of connected simple systems is a collection of homotopy classes of maps between spaces in $I_{0}$ and spaces in $J_{0}$ such that

(iv) for every $X \in I_{0}$ and $Y \in J_{0}$ the set $\Phi(X, Y)=\{[\varphi] \in[X, Y] \mid[\varphi] \in \Phi\}$ consists of a single element;

(v) if $X, \tilde{X} \in I_{0}$ and $Y, \tilde{Y} \in J_{0}$ and if $[\varphi] \in[X, Y],[f] \in \operatorname{hom}(\tilde{X}, X),[g] \in$ hom $(Y, \tilde{Y})$, then $[g \circ \varphi \circ f] \in \Phi(\tilde{X}, \tilde{Y})$.

Remarks. (i) Within a single connected simple system, the requirements on the morphisms force them to be homotopy equivalences.

(ii) If $X \in I_{0}$ and $Y \in J_{0}$, any homotopy class of maps $[\varphi] \in[X, Y]$ generates a morphism between connected simple systems.

(iii) While each connected simple system forms a category, we also have a category $\mathscr{C S S}$ whose objects are connected simple systems and whose morphisms are as in the definition.

We obtain pointed spaces from index pairs by taking quotient spaces $N / N_{0}$. The point $\hat{N}_{0}$ corresponding to $N_{0}$ is a distinguished point, so the pointed space is written as $\left(N / N_{0}, \hat{N}_{0}\right)$ or $\left(N / N_{0}, *\right)$. These pointed spaces will be the objects in a connected simple system. We obtain the morphisms hom $\left(N_{\alpha} / N_{\alpha 0}, N_{\beta} / N_{\beta 0}\right)$ as follows. If $\left(N, N_{0}\right)$ is an index pair for $S$ in $X, T \geq 0$, let $\left(N \backslash N_{0}\right)^{T}=$ $\left\{x \in X \mid x \cdot[-T, T] \subseteq N \backslash N_{0}\right\}$.

Lemma 1.8 ([12], lemma 4.7). Let $\left(N_{\alpha}, N_{\alpha 0}\right)$ and $\left(N_{\beta}, N_{\beta 0}\right)$ be index pairs for $S$ in 
$X$. There exists a $T \geq 0$ such that $\left(N_{\alpha} \backslash N_{\alpha 0}\right)^{T} \subseteq N_{\beta} \backslash N_{\beta 0}$ and $\left(N_{\beta} \backslash N_{\beta 0}\right)^{T} \subseteq N_{\alpha} \backslash N_{\alpha 0}$. Further, for every $t \geq T$, the map $\varphi_{\alpha \beta}^{\prime}: N_{\alpha} / N_{\alpha 0} \rightarrow N_{\beta} / N_{\beta 0}$ defined by

$$
\varphi_{\alpha \beta}^{\prime}([x])= \begin{cases}{[x \cdot 3 t]} & \text { if } x \cdot[0,2 t] \subseteq N_{\alpha} \backslash N_{\alpha 0}, x \cdot[t, 3 t] \subseteq N_{\beta} \backslash N_{\beta 0} \\ {\left[N_{\beta 0}\right]} & \text { otherwise }\end{cases}
$$

is continuous.

Definition 1.9. If $S$ is an isolated invariant set in $X$, the Conley index of $S$ in $X$ is the connected simple system $I(X ; S)$ whose objects are $I_{0}=\left\{N / N_{0} \mid\left(N, N_{0}\right)\right.$ is an index pair for $S$ in $X\}$ and whose morphisms are $\left[\varphi_{\alpha \beta}^{t}\right]:\left[N_{\alpha} / N_{\alpha 0}\right] \rightarrow\left[\tilde{N}_{\beta} / \tilde{N}_{\beta 0}\right]$.

THEOREM 1.10 ([12], theorem 4.10). The Conley index is a connected simple system.

That is, the quotient spaces formed from index pairs all have the same homotopy type, with the maps $\varphi^{\prime}$ providing the homotopy equivalences. A simpler form of the index, in which only the homotopy type of the index pairs is recorded, is the homotopy index $h(S)=\left[N / N_{0}, *\right]$ (cf. [1], Ch. III, § 5).

\section{Mappings in the Conley index}

In the existing theory, morphisms in $\mathscr{C} \mathscr{S} \mathscr{S}$ are used only in continuation, and appear there only as isomorphisms-morphisms between connected simple systems with the same homotopy types and the same homotopy classes of maps. To extend to more general morphisms, and so make the Conley index functional, we introduce flow maps.

Definition 2.1. Let $X$ and $Y$ be flows. A flow map is a proper continuous function $f: X \rightarrow Y$ which is equivariant with respect to the $\mathbb{R}$-actions on $X$ and $Y$ (i.e. for every $x \in X, t \in \mathbb{R}, f(x \cdot t)=f(x) \cdot t)$.

Examples of flow maps include:

(i) The inclusion $i: A \rightarrow X$ of a closed invariant (under the flow) subset of $\boldsymbol{X}$.

(ii) If $G$ is a compact group acting on a flow $X$, then multiplication by $g \in G$ and the quotient map $\pi: X \rightarrow X / G$ are flow maps, where $X / G$ is the $G$-orbit space.

(iii) In [1], Conley shows that any flow has a strongly gradient-like quotient flow, obtained by collapsing components of the chain recurrent set to points. If each component is compact, then this quotient map is a flow map.

THEOREM 2.2. Let $f: X \rightarrow Y$ be a flow map, $S \subseteq Y$ an isolated invariant set with isolating neighbourhood $N$ and (regular) index pair $\left(N_{1}, N_{0}\right)$. Then $f^{-1}(S)$ is an isolated invariant set with isolating neighbourhood $f^{-1}(N)$ and (regular) index pair $\left(f^{-1}\left(N_{1}\right), f^{-1}\left(N_{0}\right)\right)$.

Proof. Invariance under the flow is preserved by pull-backs, so $f^{-1}(S)$ is invariant. Further, if $x \cdot \mathbb{R} \subseteq f^{-1}(N)$, then $f(x) \cdot \mathbb{R}=f(x \cdot \mathbb{R}) \subseteq N$ and $f(x) \subseteq I(N)=S$. The map $f$ is proper, so $f^{-1}(N)$ is compact, and so is an isolating neighbourhood for isolated invariant set $f^{-1}(S)$.

Likewise, $\quad \operatorname{cl}_{\gamma}\left(N_{1} \backslash N_{0}\right)$ is an isolating neighbourhood for $S$, so $\mathrm{cl}_{X}\left(f^{-1}\left(N_{1}\right) \backslash f^{-1}\left(N_{0}\right)\right)=f^{-1}\left(\operatorname{cl}_{Y}\left(N_{1} \backslash N_{0}\right)\right)$ is an isolating neighbourhood for 
$f^{-1}(S)$. Positive invariance is also preserved by pull-backs, so $f^{-1}\left(N_{0}\right)$ is positively invariant in $f^{-1}\left(N_{1}\right)$. If $x \in f^{-1}\left(N_{1}\right)$ so that $x \cdot R^{+} \not \subset f^{-1}\left(N_{1}\right)$, then $f(x) \subseteq N_{1}$ with $f(x) \cdot \mathbb{R} \not \subset N_{1}$. There is then a $t \geq 0$ so that $f(x) \cdot t \in N_{0}$ and $f(x) \cdot[0, t] \subseteq N_{1}$. That is, $x \cdot t \in f^{-1}\left(N_{0}\right)$ and $x \cdot[0, t] \subseteq f^{-1}\left(N_{1}\right)$, and $f^{-1}\left(N_{0}\right)$ is an exit set for $f^{-1}\left(N_{1}\right)$.

Finally, if $\left(N_{1}, N_{0}\right)$ is a regular index pair, then the exit-time map $\tau_{+}: N_{1} \rightarrow \mathbb{R}$ is continuous. But $\tau_{+} \circ f: f^{-1}\left(N_{1}\right) \rightarrow \mathbb{R}$ is then the exit-time map for $\left(f^{-1}\left(N_{1}\right), f^{-1}\left(N_{0}\right)\right)$, and so it is a regular index pair also.

Definition 2.3. Suppose $f: X \rightarrow Y$ is flow map, $S \subseteq Y$ is an isolated invariant set and $\left(N_{1}, N_{0}\right)$ is an index pair for $S$ in $Y$. Then $h(f): h\left(f^{-1}(S)\right) \rightarrow h(S)$ is the homotopy class $[f]:\left[f^{-1}\left(N_{1}\right) / f^{-1}\left(N_{0}\right)\right] \rightarrow\left[N_{1} / N_{0}\right]$ and $I(f): I\left(X ; f^{-1}(S)\right) \rightarrow I(X ; S)$ is the morphism of connected simple systems induced by $h(f)$.

Proposition 2.4. $I(f)$ is a morphism of connected simple systems.

Proof. Let $\left(N_{1}, N_{0}\right)$ and $\left(\tilde{N}_{1}, \tilde{N}_{0}\right)$ be index pairs for $S$ in $Y$. Using the notation of lemma 1.8, choose $T>0$ so that $\left(N_{1} \backslash N_{0}\right)^{T} \subseteq \tilde{N}_{1} \backslash \tilde{N}_{0}$ and $\left(\tilde{N}_{1} \backslash \tilde{N}_{0}\right)^{T} \subseteq N_{1} \backslash N_{0}$.

(i) As $\left(f^{-1}\left(N_{0} \backslash N_{0}\right)\right)^{T}=f^{-1}\left(\left(N_{1} \backslash N_{0}\right)^{T}\right),\left(f^{-1}\left(N_{1} \backslash N_{0}\right)\right)^{T} \subseteq f^{-1}\left(\tilde{N}_{1} \backslash \tilde{N}_{0}\right)$. Thus, if $\varphi_{Y}^{\prime}$ represents the homotopy class hom $\left(N_{1} / N_{0}, \tilde{N}_{1} / \tilde{N}_{0}\right)$ in $I(S)$, then

$$
\varphi_{X}^{\prime}([x])= \begin{cases}{[x \cdot 3 t]} & \text { if } x \cdot[0,2 t] \subseteq f^{-1}\left(N_{1} \backslash N_{0}\right), x \cdot[t, 3 t] \subseteq f^{-1}\left(\tilde{N}_{1} \backslash \tilde{N}_{0}\right) \\ {\left[\tilde{N}_{0}\right]} & \text { otherwise }\end{cases}
$$

is continuous for every $t>T$ and represents the homotopy class

$$
\operatorname{hom}\left(f^{-1}\left(N_{1}\right) / f^{-1}\left(N_{0}\right), f^{-1}\left(\tilde{N}_{1}\right) / f^{-1}\left(\tilde{N}_{0}\right)\right) \text { in } I\left(X ; f^{-1}(S)\right) \text {. }
$$

(ii) $I(f)$ will be a well defined morphism of connected simple systems provided $\varphi_{Y}^{t} \circ I(f)=I(f) \circ \varphi_{X}^{t}$ :

$$
\varphi_{Y}^{t} \circ I(f)[x]= \begin{cases}{[f(x) \cdot 3 t]} & \text { if } f(x) \cdot[0,2 t] \subseteq N_{1} \backslash N_{0}, f(x) \cdot[t, 3 t] \subseteq \tilde{N}_{1} \backslash \tilde{N}_{0}, \\ {\left[\tilde{N}_{0}\right]} & \text { otherwise, }\end{cases}
$$

while

$$
I(f) \circ \varphi_{X}^{\prime}[x]= \begin{cases}{[f(x \cdot 3 t)]} & \text { if } x \cdot[0,2 t] \subseteq f^{-1}\left(N_{1} \backslash N_{0}\right), x \cdot[t, 3 t] \subseteq f^{-1}\left(\tilde{N}_{1} \backslash \tilde{N}_{0}\right), \\ {\left[\tilde{N}_{0}\right]} & \text { otherwise. }\end{cases}
$$

As $f$ commutes with the quotients and the flows, the two formulae agree.

We are now free to study the role of flow maps in either the homotopy index $h(S)$ or the Conley index $I(X ; S)$. As connected simple systems give more precise information than homotopy classes, we will limit our attention to the Conley index.

Proposition 2.5. Given a commutative diagram of flow maps

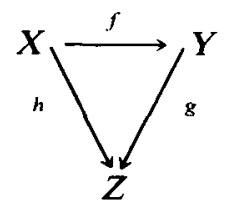


and an isolated invariant set $S \subseteq Z$, then there exists a commutative diagram of connected simple systems

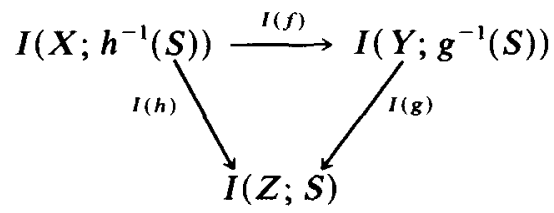

Proof. This is immediate from the formulae in 2.4 .

We next want to describe how flow maps behave under continuation. To do so, some results on isolating neighbourhoods are needed.

LEMMA 2.6. Let $A \subseteq X$ be a closed pair of flows and $S \subseteq X$ an isolated invariant set. Let $N \subseteq A$ be an isolating neighbourhood for $S \cap A$ in $A$. Then there exists an isolating neighbourhood $M$ for $S$ in $X$ such that $M \cap A=N$.

Proof. The inclusion $i: A \rightarrow X$ is a flow map, so $i^{-1}(S)=S \cap A$ is isolated in $A$. Choose an isolating neighbourhood $\tilde{M}$ for $S$ in $X$. $\tilde{M} \cap A \cap N$ is an isolating neighbourhood for $S \cap A$ in $A$, so $S \cap A \subseteq \operatorname{int}_{A}(\tilde{M} \cap A \cap N)$. Let $U=\tilde{M} \cap(A \backslash N)$. Then $S$ and $\operatorname{cl}_{\tilde{M} \cap A}(U)=\operatorname{cl}_{\tilde{M}}(U)$ are disjoint closed subsets of $\tilde{M}$. Choose disjoint open sets $V_{1}, V_{2}$ in $\tilde{M}$ with $\operatorname{cl}_{\dot{M}}(U) \subseteq V_{1}$ and $S \subseteq V_{2}$, and let $M=\left(\tilde{M} \backslash V_{1}\right) \cup N$.

(i) $\boldsymbol{M} \cap A=\left(\left(\tilde{M} \backslash V_{1}\right) \cap A\right) \cup N$, with $\left(\left(\tilde{M} \backslash V_{1}\right) \cap A\right) \subseteq((\tilde{M} \backslash U) \cap A)=\tilde{M} \cap N$, so $M \cap A=N$.

(ii) $S \subseteq V_{2} \subseteq \tilde{M} \backslash V_{1} \subseteq M$ and $S \subseteq$ int $_{X} \tilde{M}$, so $S \subseteq$ int $_{X} M$.

(iii) $N \subseteq A$, which is invariant. Thus orbits in $\tilde{M} \backslash V_{1}$ can intersect $N$ only if they are contained in $A$, hence in $\left(\tilde{M} \backslash V_{1}\right) \cap A \subseteq N$. Thus $I(M)=I\left(\left(\tilde{M} \backslash V_{1}\right) \cup N\right)=$ $I\left(\tilde{M} \backslash V_{1}\right) \cup I(N) \subseteq I(\tilde{M}) \cup I(N) . I(\tilde{M})=S$ and $I(N)=S \cap A$, so $I(M) \subseteq S$. But $S \subseteq M$, so $I(M)=S$ and $M$ is an isolating neighbourhood for $S$ in $X$.

LEMmA 2.7. Let $f: X \rightarrow Y$ be a flow map, $S \subseteq Y$ an isolated invariant set and $N \subseteq X$ an isolating neighbourhood for $f^{-1}(S)$. Then there exists an isolating neighbourhood $M$ of $S$ in $Y$ such that $f^{-1}(M) \subseteq$ int $_{X} N$.

Proof. (i) First assume $f$ is onto. By taking one-point compactifications if necessary, we may assume that $X$ and $Y$ are compact.

Let $V=\left\{y \in Y \mid f^{-1}(y) \subseteq\right.$ int $\left._{X} N\right\}$. Then $Y \backslash V=f\left(X \backslash\right.$ int $\left._{X} N\right)$ is compact and $V$ is open. As $f^{-1}(S) \subseteq$ int $_{X} N, S \subseteq V$. If $N=X$, the result is trivial. If $N \neq X$, then $X \backslash$ int $_{X} N \neq \varnothing$ and $Y \backslash V \neq \varnothing$.

If $S=\varnothing$, take $M=\varnothing$. If $S \neq \varnothing$, choose $\alpha: Y \rightarrow[0,1]$ so that $\alpha(S)=0, \alpha(Y \backslash V)=1$. Then $(\alpha \circ f)\left(X \backslash\right.$ int $\left._{X} N\right)=\alpha(Y \backslash V)=1$ is closed in [0,1]. Choose $0<\varepsilon<1$ and let $M_{1}=\alpha^{-1}[0, \varepsilon]$. Then $S \subseteq \alpha^{-1}(0) \subseteq$ int $_{Y} M_{1}$. (On the one-point compactifications, $f^{-1}\left(\infty_{Y}\right)=\left\{\infty_{X}\right\} \not \subset$ int $_{X} N$, so $\infty_{Y} \in Y \backslash V$ and $\infty_{X} \notin M_{1}$.) Let $M_{2}$ be an isolating neighbourhood for $S$ in $Y$ and let $M=M_{1} \cap M_{2}$. Then $S \subseteq$ int $_{Y} M_{1} \cap$ int $_{Y} M_{2} \subseteq$ int $_{Y} M$ and $S \subseteq I\left(M_{1}\right) \cap I\left(M_{2}\right)=I(M) \subseteq I\left(M_{2}\right)=S$, so $M$ is an isolating neighbourhood for $S$. Further, $f^{-1}(M) \subseteq f^{-1}\left(M_{1}\right) \subseteq$ int $_{X} N$.

(ii) If $f$ is not onto, choose an isolating neighbourhood $M^{\prime}$ for $f(x) \cap S$ in $f(X)$ with $f^{-1}\left(M^{\prime}\right) \subseteq$ int $_{X} N$. By lemma 2.6, $M^{\prime}$ then extends to an isolating neighbourhood $M$ for $S$ in $Y$ with $f(X) \cap M=M^{\prime}$, hence with $f^{-1}(M)=f^{-1}\left(M^{\prime}\right) \subseteq$ int $_{X} N$.

Recall that, if $Y \times \Lambda$ is a parametrized family of flows, we define $\mathscr{S}(Y)=$ $\left\{\left(S_{\lambda}, \lambda\right) \mid S_{\lambda}\right.$ is an isolated invariant set in $\left.Y_{\lambda}\right\}$. We write $U_{\lambda}$ for $U$ with the $\lambda$-flow, 
and $I_{\lambda}(U)$ for the maximal invariant set of $U$ in the $\lambda$-flow. If $F: X \times \Lambda \rightarrow Y \times \Lambda$ is a flow map, define $\mathscr{S}(F): \mathscr{S}(Y) \rightarrow \mathscr{S}(X)$ by $\mathscr{S}(F)\left(S_{\lambda}, \lambda\right)=\left(F_{\lambda}^{-1}\left(S_{\lambda}\right), \lambda\right)$.

THEOREM 2.8. If $F: X \times \Lambda \rightarrow Y \times \Lambda$ is a flow map, then $\mathscr{S}(F)$ is continuous.

Proof. Bases for the topologies of $\mathscr{S}(X)$ and $\mathscr{S}(Y)$ are defined as follows. Let $N \subseteq Y$ be compact and let $\Lambda(N)$ be the set of $\lambda \in \Lambda$ for which $N$ is an isolating neighbourhood. $\Lambda(N)$ is open, and we define $\sigma_{N}: \Lambda(N) \rightarrow \mathscr{S}(Y)$ by $\sigma_{N}(\lambda)=\left(I_{\lambda}(N), \lambda\right)$. Then $\mathscr{S}(Y)$ has basis $B(Y)=\left\{\sigma_{N}(U) \mid N \subseteq Y\right.$ is compact, $U \subseteq \Lambda(N)$ is open $\}$. Let $\sigma_{N}(U)$ be such a basis element in $\mathscr{S}(X)$. Then $\mathscr{S}(F)^{-1}\left(\sigma_{N}(U)\right)=\left\{\left(S_{\lambda}, \lambda\right) \mid \lambda \in U, S_{\lambda}\right.$ an isolated invariant set, $\left.F_{\lambda}^{-1}\left(S_{\lambda}\right)=I_{\lambda}(N)\right\}$. By lemma 2.7 , for every such $\left(S_{\lambda}, \lambda\right)$ there exists a compact $M_{\lambda} \subseteq Y_{\lambda}$ with $\lambda \in \Lambda\left(M_{\lambda}\right)$ and $S_{\lambda}=I_{\lambda}\left(M_{\lambda}\right)$.

LEMMA 2.9. For every $M_{\lambda}$ there exists an open $\Lambda$-neighbourhood $U\left(M_{\lambda}\right)$ of $\lambda$ contained in $U \cap \Lambda\left(M_{\lambda}\right)$ with $I_{\mu}\left(F_{\mu}^{-1}\left(M_{\lambda}\right)\right)=I_{\mu}(N)$ for every $\mu \in U\left(M_{\lambda}\right)$.

Proof. It suffices to show that there exist open $\Lambda$-neighbourhoods $V\left(M_{\lambda}\right)$ and $W\left(M_{\lambda}\right)$ of $\lambda$ in $\Lambda$ with $I_{\mu}\left(F_{\mu}^{-1}\left(M_{\lambda}\right)\right) \subseteq I_{\mu}(N)$ for all $\mu \in V\left(M_{\lambda}\right)$ and $I_{\mu}(N) \subseteq I_{\mu}\left(F_{\mu}^{-1}\left(M_{\lambda}\right)\right)$ for all $\mu \in W\left(M_{\lambda}\right)$. Then

$$
U\left(M_{\lambda}\right)=U \cap \Lambda\left(M_{\lambda}\right) \cap V\left(M_{\lambda}\right) \cap W\left(M_{\lambda}\right) .
$$

$\Lambda$ is locally compact, so there exists a compact $C_{\lambda} \subseteq \Lambda$ with $\lambda \in \operatorname{int}_{\Lambda}\left(C_{\lambda}\right) . F^{-1}\left(M_{\lambda} \times\right.$ $\left.C_{\lambda}\right)$ is compact, as $F$ is proper. Let $\pi_{i}$ be the projection of $X \times \Lambda$ onto its $i$ th factor. Let $Z=N \cup \pi_{1}\left(F^{-1}\left(M_{\lambda} \times C_{\lambda}\right)\right)$ and consider $\left(F \mid Z \times C_{\lambda}\right): Z \times C_{\lambda} \rightarrow Y \times C_{\lambda}$. $F^{-1}\left(M_{\lambda} \times C_{\lambda}\right)$ and $\left(Z \backslash\right.$ int $\left._{Z} N\right) \times C_{\lambda}$ are closed in $Z \times C_{\lambda}$, so

$$
\left\{\mu \in C_{\lambda} \mid F_{\mu}^{-1}\left(M_{\lambda}\right) \cap\left(Z \backslash \text { int }_{Z} N\right) \neq \varnothing\right\}=\pi_{2}\left(F^{-1}\left(M_{\lambda} \times C_{\lambda}\right) \cap\left\{\left(Z \backslash \text { int }_{Z} N\right) \times C_{\lambda}\right\}\right)
$$

is closed. Then

$$
\left\{\mu \in C_{\lambda} \mid F^{-1}\left(M_{\lambda}\right) \cap\left(Z \backslash \text { int }_{Z} N\right)=\varnothing\right\}=\left\{\mu \in C_{\lambda} \mid F_{\mu}^{-1}\left(M_{\lambda}\right) \subseteq \text { int }_{Z} N\right\}
$$

is open in $C_{\lambda}$, and $V\left(M_{\lambda}\right)=\left\{\mu \in\right.$ int $_{\Lambda} C_{\lambda} \mid F_{\mu}^{-1}\left(M_{\lambda}\right) \subseteq$ int $\left._{Z} N\right\}$ is an open neighbourhood of $\lambda$ in $\Lambda$. Clearly, if $\mu \in V\left(M_{\lambda}\right)$, then $I_{\mu}\left(F_{\mu}^{-1}\left(M_{\lambda}\right)\right) \subseteq I_{\mu}(N)$.

$W\left(M_{\lambda}\right)$ is constructed analogously. $\left(F \mid Z \times C_{\lambda}\right)^{-1}$ (int $\left.M_{Y} M_{\lambda}\right)$ is open in $Z \times C_{\lambda}$, and $I\left(N \times C_{\lambda}\right)$ is closed in $Z \times C_{\lambda}$. Analogous to the above argument, $\left\{\mu \in C_{\lambda} \mid I_{\mu}(N) \subseteq F_{\mu}^{-1}\left(\right.\right.$ int $\left.\left._{Y} M_{\lambda}\right)\right\}$ is open in $C_{\lambda}$ and contains $\lambda$.

$$
W\left(M_{\lambda}\right)=\left\{\mu \in \operatorname{int}_{\Lambda} C_{\lambda} \mid I_{\mu}(N) \subseteq F_{\mu}^{-1}\left(\operatorname{int}_{Y} M_{\lambda}\right)\right\}
$$

then has the required properties.

To complete the theorem proof, we must show that $\mathscr{S}(F)^{-1}\left(\sigma_{N}(U)\right)$ is open in $\mathscr{S}(Y)$. For every element of $\mathscr{S}(F)^{-1}\left(\sigma_{N}(U)\right)$, choose an $M_{\lambda}$ and a $U\left(M_{\lambda}\right)$ as in 2.7 and 2.9. Each $\sigma_{M}\left(U\left(M_{\lambda}\right)\right)$ is a basis element of $\mathscr{S}(Y)$, so $\bigcup \sigma_{M}\left(U\left(M_{\lambda}\right)\right)$ is open in $\mathscr{S}(Y)$. By construction, $\mathscr{S}(F)^{-1}\left(\sigma_{N}(U)\right)$ is contained in this union, so we need only show that each $\sigma_{M}\left(U\left(M_{\lambda}\right)\right) \subseteq \mathscr{S}(F)^{-1}\left(\sigma_{N}(U)\right)$. If $\mu \in U\left(M_{\lambda}\right)$, then $\sigma_{M}(\mu)=$ $\left(I_{\mu}\left(M_{\lambda}\right), \mu\right)$ and $\mathscr{S}(F)\left(\sigma_{M}(\mu)\right)=\left(I_{\mu}(N), \mu\right)$ by lemma 2.9, and $\sigma_{M}(\mu) \epsilon$ $\mathscr{S}(F)^{-1}\left(\sigma_{N}(U)\right)$.

THEOREM 2.10. If $F: X \times \Lambda \rightarrow Y \times \Lambda$ is a flow map of parametrized families of flows 
with $\left(S_{\lambda}, \lambda\right)$ and $\left(S_{\mu}, \mu\right) \in \mathscr{P}(Y)$ related by continuation, then $\left(F_{\lambda}^{-1}\left(S_{\lambda}\right), \lambda\right)$ and $\left(F_{\mu}^{-1}\left(S_{\mu}\right), \mu\right) \in \mathscr{S}(X)$ are related by continuation, and the diagram commutes:

$$
\begin{array}{lr}
I\left(X_{\lambda} ; F_{\lambda}^{-1}\left(S_{\lambda}\right)\right) \stackrel{I\left(F_{\lambda}\right)}{\longrightarrow} I\left(Y_{\lambda} ; S_{\lambda}\right) \\
F_{X}(\lambda, \mu) \mid= & \cong F_{Y}(\lambda, \mu) \\
I\left(X_{\mu} ; F_{\mu}^{-1}\left(S_{\mu}\right)\right) \stackrel{I\left(F_{\mu}\right)}{\longrightarrow} I\left(Y_{\mu} ; S_{\mu}\right)
\end{array}
$$

Proof. $\left(S_{\lambda}, \lambda\right)$ and $\left(S_{\mu}, \mu\right)$ are related by continuation if and only if there exists a path $\omega:[0,1] \rightarrow \mathscr{P}(Y)$ with $\omega(0)=\left(S_{\lambda}, \lambda\right)$ and $\omega(1)=\left(S_{\mu}, \mu\right)$. Then $\mathscr{S}(F) \circ \omega:[0,1] \rightarrow \mathscr{S}(X)$ is a path with endpoints $\left(F_{\lambda}^{-1}\left(S_{\lambda}\right), \lambda\right)$ and $\left(F_{\mu}^{-1}\left(S_{\mu}\right), \mu\right)$. The continuation theorem ([12], theorem 6.7) states that if $\left(S_{\lambda}, \lambda\right),\left(S_{\mu}, \mu\right) \in \mathscr{S}(Y)$ are related by continuation, then there exist isomorphisms of connected simple systems $F_{Y}(\lambda, \mu): I\left(Y_{\lambda} ; S_{\lambda}\right) \leftrightarrows I\left(Y_{\mu} ; S_{\mu}\right): F_{Y}(\mu, \lambda)$ which are independent of the path in $\mathscr{S}(Y)$ relating $\left(S_{\lambda}, \lambda\right)$ and $\left(S_{\mu}, \mu\right)$. The morphism $F_{Y}(\lambda, \mu)$ is constructed by covering $[0,1]$ with intervals $K_{1}$ and taking a sequence $0=s_{0}, s_{1}, \ldots, s_{n}=1$ so that $s_{i} \in K_{i} \cap K_{i+1}$. These $K$ may be chosen so that the inclusion-induced morphism $j_{Y}(\lambda, K): I\left(Y_{\lambda} ; S_{\lambda}, \lambda\right) \rightarrow I\left(Y \times K ; S_{K}\right)$ is a homotopy equivalence for every $\lambda \in K$, where $S_{K}=\bigcup\left\{\left(S_{\mu}, \mu\right) \mid \mu \in \omega(K)\right\}$. The homotopy inverse of $j_{Y}(\lambda, K)$ is denoted $F_{Y}(\lambda, K)$. We then take

$$
F_{Y}(\lambda, \mu)=F_{Y}\left(\lambda_{n}, K_{n}\right) \circ j_{Y}\left(\lambda_{n-1}, K_{n}\right) \circ \cdots \circ F_{Y}\left(\lambda_{1}, K_{1}\right) \circ j_{Y}\left(\lambda_{0}, K_{1}\right),
$$

where $\lambda_{i}=\omega\left(s_{i}\right)$.

We obtain a common set of the $K$ for $X$ and $Y$ by taking covers $\left\{K_{j}(X)\right\}$ and $\left\{K_{k}(Y)\right\}$ which are admissible for $X$ and $Y$ respectively, and taking $K_{j k}=K_{j}(X) \cap$ $K_{k}(Y)$. Take connected components of the $K_{j k}$ and order them so that $K_{i} \cap K_{i+1} \neq \varnothing$. As $j\left(\lambda_{i}, K_{i+1}\right)$ is inclusion-induced, hence flow map-induced, we have $I\left(F_{\omega(K)}\right) \circ j_{X}(\lambda, K)=j_{Y}(\lambda, K) \circ I\left(F_{\lambda}\right)$ from proposition 2.5. As $F(\lambda, K)$ is the homotopy inverse of $j(\lambda, K)$, a simple diagram chase shows that $I\left(F_{\omega(K)}\right) \circ F_{X}(\lambda, K)=F_{Y}(\lambda, K) \circ I\left(F_{\lambda}\right)$.

Definition 2.11. Flow maps $f, g: X \rightarrow Y$ are homotopic as flow maps if there exists a flow map $F: X \times[0,1] \rightarrow Y \times[0,1]$ with $F_{0}=f, F_{1}=g$, where $X \times[0,1]$ and $Y \times[0,1]$ have product parametrization (i.e. the flows on $X$ and $Y$ are independent of the parameter value).

Corollary 2.12. If $f, g: X \rightarrow Y$ are homotopic as flow maps, then for every isolated invariant set $S \subseteq Y, f^{-1}(S)=g^{-1}(S)$ and $I(f)=I(g): I\left(X f^{-1}(S)\right) \rightarrow I(Y ; S)$.

Proof. In a product parametrization, $\left(S_{\lambda}, \lambda\right)$ and $\left(S_{\mu}, \mu\right)$ are related by continuation if and only if $S_{\lambda}=S_{\mu}$.

Example 2.13. The flow on the torus $T^{2}$ given by $\mathscr{Y}_{0}(\dot{\theta}, \dot{\phi})=(\sin (\theta) \cos (\phi), \sin (\phi))$ has hyperbolic critical points $x_{0}=(0, \pi), x_{1}=(\pi, \pi), x_{2}=(\pi, 0), x_{3}=(0,0)$ with indices $I_{0}\left(T^{2} ; x_{0}\right)=\Sigma^{0}, I_{0}\left(T^{2} ; x_{1}\right)=\Sigma^{1}, I_{0}\left(T^{2} ; x_{2}\right)=\Sigma^{1}, I_{0}\left(T^{2} ; x_{3}\right)=\Sigma^{2}$, and with two connecting orbits from $(\pi, 0)$ to $(0, \pi)$. These connecting orbits can be perturbed off by continuing to the flow given by $\mathscr{Y}_{1}(\dot{\theta}, \dot{\phi})=(\sin (\theta), \sin (\phi))$. This flow has 
$x_{0}, x_{1}, x_{2}, x_{3}$ isolated with indices $I_{1}\left(T^{2} ; x_{0}\right)=\Sigma^{1}, I_{1}\left(T^{2} ; x_{1}\right)=\Sigma^{0}, I_{1}\left(T^{2} ; x_{2}\right)=\Sigma^{1}$, $I_{1}\left(T^{2} ; x_{3}\right)=\Sigma^{2}$. The continuation can be done so that $x_{0}$ in the $\mathscr{Y}_{0}$ flow and $x_{1}$ in the $\mathscr{Y}_{1}$ flow are related by continuation (and vice versa).

Each of these flows lifts to a flow on $X=[-\pi, \pi] \times[-\pi, \pi]$. That is, define $\mathscr{X}_{0}(x, y)=(\sin (x) \cos (y), \sin (y))$ and $\mathscr{X}_{1}(x, y)=(\sin (x), \sin (y))$. Then the map $f(x, y)=(\theta, \phi)$ is a flow map at 0 and at 1 . Further, $\mathscr{X}_{0}$ continues to $\mathscr{X}_{1}$. However, there is no flow map $F: X \times[0,1] \rightarrow Y \times[0,1]$ with $F_{0}=F_{1}=f$, for $x_{0}$ in the $\mathscr{Y}_{0}$ flow and $x_{1}$ in the $\mathscr{Y}_{1}$ flow are related by continuation, but $I_{0}\left(X ; f^{-1}\left(x_{0}\right)\right)=\Sigma^{0} \vee \Sigma^{0}$, while $I_{1}\left(X ; f^{-1}\left(x_{1}\right)\right)=\Sigma^{0} \vee \Sigma^{0} \vee \Sigma^{0} \vee \Sigma^{0}$. If such a flow map $F$ existed, then theorem 2.10 would require $I_{0}\left(X ; f^{-1}\left(x_{0}\right)\right) \cong I_{1}\left(X ; f^{-1}\left(x_{1}\right)\right)$.

\section{Functorial constructions}

A given invariant set may be isolated in more than one flow, and will in general have a different index when viewed in different flows. One way in which the flow may be changed is to restrict to some subflow. This may be done via flow maps. If $A \subseteq X$ is a closed invariant subspace, the inclusion map $i: A \rightarrow X$ is a flow map, with $i^{-1}(S)=S \cap A$. With this, we will be able to define a category of isolated invariant sets and flow maps and a homology functor on this category. That is, since inclusions of closed subflows will lie in the category, we will be able to give it the 'pair' structure needed for a homology theory.

Definition 3.1. The category $\mathscr{I S}$ of isolated invariant sets has objects of the form $(X, A ; S)$, where $X$ is a locally compact metric flow, $A \subseteq X$ is a closed invariant subspace and $S \subseteq X$ is an isolated invariant set in $X$. Morphisms are flow maps $f:(X, A ; S) \rightarrow(Y, B ; T)$ with $f(A) \subseteq B$ and $f^{-1}(T)=S$.

Remarks. (i) $\mathscr{I S}$ can readily be checked to be a category. Its pair nature lies in the fact that $(X, A)$ is a pair of flows. Properly, $(X, A ; S)$ is to be regarded as a pair of isolated invariant sets in a pair of flows, with $S \cap A$ the isolated invariant set in $A$. A single isolated invariant set in a single flow may be obtained by taking $A=\varnothing$.

(ii) Everything above can be done with local flows or discrete dynamical systems instead of flows. If $X, Y$ local flows, define a local flow map to be a proper continuous map with the following equivariance properties:

(a) if $x \in X, t \in \mathbb{R}$ such that $x \cdot t \in X$, then $f(x) \cdot t \in Y$ and $f(x) \cdot t=f(x \cdot t)$;

(b) if $x \in X, t \in \mathbb{R}^{+}$such that $f(x) \cdot t \in Y$, then $x \cdot t \in X$.

If $f^{-1}(S)$ is replaced by $f^{-1}(S) \cap I(X)$, all of the theorems above remain valid. We can then define a category $\mathscr{L} \mathscr{Y} \mathscr{S}$ of isolated invariant sets in local flows.

If $X, Y$ have discrete dynamical systems given by homeomorphisms $g$ and $h$ respectively, a discrete flow map is a proper continuous map $f: X \rightarrow Y$ such that $f \circ g=h \circ f$. If the Conley index is replaced by the shape index defined in [11], all of the theorems above remain valid. For the present, however, we will limit ourselves to the category $\mathscr{S} \mathscr{P}$ for continuous flows.

By taking pairs of spaces and maps of pairs, the definitions of a connected simple system and a morphism of connected simple systems have obvious relative category 
versions. We now use $\mathscr{C} \mathscr{S} \mathscr{S}$ to denote this category, and view the single space category as a sub-category. With this relative category, we can define:

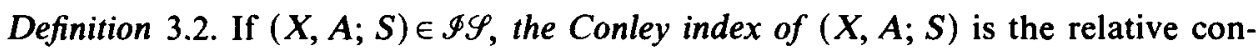
nected simple system $I(X, A ; S)$ whose objects are

$$
I_{0}=\left\{\left(N / N_{0},(N \cap A) /\left(N_{0} \cap A\right)\right) \mid\left(N, N_{0}\right) \text { is an index pair for } S \text { in } X\right\}
$$

and whose morphisms are

$$
\left[\varphi^{\prime}\right]:\left(N / N_{0},(N \cap A) \backslash\left(N_{0} \cap A\right)\right) \rightarrow\left(\tilde{N} / \tilde{N}_{0},(\tilde{N} \cap A) /\left(\tilde{N}_{0} \cap A\right)\right) .
$$

If $f:(X, A ; S) \rightarrow(Y, B ; T)$ is a morphism in $\mathscr{Y}, I(f)$ is the morphism of relative connected simple systems induced by

$$
[f]:\left[f^{-1}\left(N / N_{0}\right), f^{-1}\left((N \cap A) /\left(N_{0} \cap A\right)\right)\right] \rightarrow\left[N / N_{0},(N \cap A) /\left(N_{0} \cap A\right)\right] .
$$

To emphasize the functorial dependence of the index on the flow, we write the Conley index as $I(X, A ; S)$ (or as $I(X ; S)$ when $A=\varnothing)$ rather than $I(S)$ or $I(S, X)$. This differs from the notation of other authors, as will the notation for the homology Conley index. With this, we have the following reformulation of the Conley index:

Proposition 3.3. The Conley index $I$ is a functor from the category $\mathscr{I} \mathscr{S}$ to the category $\mathscr{C} \mathscr{S}$.

To pass to the homology Conley index, we note the following:

Proposition 3.4. For every $(X, A ; S) \in \mathscr{G}$, the connected simple system $I(X, A ; S)$ is an inverse system of sets.

Proof. If $\left(N_{1 \alpha}, N_{0 \alpha}\right)$ and $\left(N_{1 \beta}, N_{0 \beta}\right)$ are index pairs for $S$ in $X$, then

$$
\left(N_{1 \alpha} \cap N_{1 \beta},\left(N_{1 \alpha} \cap N_{0 \beta}\right) \cup\left(N_{0 \alpha} \cap N_{1 \beta}\right)\right)
$$

is an index pair. Define a partial order on $I(X, A ; S)$ by $\left[N_{1 \alpha} / N_{0 \alpha}\right] \leq\left[N_{1 \beta} / N_{0 \beta}\right]$ if $N_{1 \beta} \backslash N_{0 \beta} \subseteq N_{1 \alpha} \backslash N_{0 \alpha}$. Then for every $\alpha, \beta$ there exists a $\gamma$ such that $\alpha, \beta \leq \gamma$. If $\alpha \leq \beta \leq \gamma$, then $N_{1 \gamma} \backslash N_{0 \gamma} \subseteq N_{1 \beta} \backslash N_{0 \beta} \subseteq N_{1 \alpha} \backslash N_{0 \alpha}$, so $\leq$ is a partial order. Further, the maps $\left[\varphi_{\alpha, \beta}^{1}\right]: N_{1 \alpha} / N_{0 \alpha} \rightarrow N_{1 \beta} / N_{0 \beta}$ of the connected simple system satisfy

$$
\left[\varphi_{\alpha, \alpha}^{\prime}\right]=[\mathrm{id}] \text { and }\left[\varphi_{\alpha, \gamma}^{t+s}\right]=\left[\varphi_{\alpha, \beta}^{t} \circ \varphi_{\beta, \gamma}^{s}\right]=\left[\varphi_{\alpha, \beta}^{t}\right] \circ\left[\varphi_{\beta, \gamma}^{s}\right] \text { for all } \alpha \leq \beta \leq \gamma \text {. }
$$

Thus, if we apply the singular homology $H_{*}$ to a connected simple system, we obtain an inverse system of graded groups, with all of the groups isomorphic and all of the maps between them isomorphisms. We can then take the inverse limit to obtain a single graded group. This is equivalent to making a consistent choice of generators for the groups $H_{*}\left(N_{1} / N_{0}, *\right)$.

Definition 3.5. The homology Conley index is the functor $C H_{*} \equiv \varliminf^{\lim } \circ H_{*} \circ I$ from the category $\mathscr{I S}$ to the category of graded Abelian groups.

We will denote $\mathrm{CH}_{*}(X, \varnothing ; S)$ by $\mathrm{CH}_{*}(X ; S)$ and $C H_{*}(f)$ by $f_{*}$. If $S$ is a compact flow, then $S$ is isolated in $S$, and $(S, \varnothing ; S) \in \mathscr{I} \mathscr{S} . S$ has only one index pair in itself, namely $(S, \varnothing)$, so $\mathrm{CH}_{*}(S ; S)=H_{*}(S)$, the singular homology of $S$. By taking trivial flows if necessary, any compact metric space can viewed as a compact flow, and any map between compact metric spaces can be viewed as a flow map. 
THEOREM 3.6. $\mathrm{CH}_{*}$ is a homology functor on $\mathscr{\Phi} \mathscr{S}$ in the following sense:

(i) if $\mathrm{id}:(X, A ; S) \rightarrow(X, A ; S)$ is the identity map, then $\mathrm{id}_{*}: C H_{*}(X, A ; S) \rightarrow$ $\mathrm{CH}_{*}(X, A ; S)$ is the identity homomorphism;

(ii) if $f:(X, A ; R) \rightarrow(Y, B ; S)$ and $g:(\dot{Y}, B ; S) \rightarrow(Z, C ; T)$ are morphisms in $\mathscr{Y}$, then $(g \circ f)_{*}=g_{*} \circ f_{*}$;

(iii) if $i:(A, \varnothing ; A \cap S) \rightarrow(X, \varnothing ; S)$ and $j:(X, \varnothing ; S) \rightarrow(X, A ; S)$ are inclusion maps, then there exists a degree -1 map $\partial: \mathrm{CH}_{*}(X, A ; S) \rightarrow C H_{*}(A ; A \cap S)$ so that the sequence is exact:

$$
\cdots \stackrel{\partial}{\longrightarrow} \mathrm{CH}_{n}(A ; A \cap S) \stackrel{i_{*}}{\longrightarrow} \mathrm{CH}_{n}(X ; S) \stackrel{j_{*}}{\longrightarrow} \mathrm{CH}_{n}(X, A ; S) \rightarrow \cdots ;
$$

(iv) if $(X, A ; S) \rightarrow(Y, B ; T)$ is a morphism in $\Phi \mathscr{S}$, then $\partial \circ f_{*}=(f \mid A)_{*}{ }^{\circ} \partial$;

(v) if $f, g:(X, A ; S) \rightarrow(Y, B ; T)$ are homotopic as flow maps, then $f_{*}=g_{*}$;

(vi) if $(X, A, S) \in \mathscr{S}$ and $U \subseteq A$ is an open invariant set with $\operatorname{cl}_{X} U \subseteq$ int $_{X} A$, then the inclusion $i:(X \backslash U, A \backslash U ; S \cap(X \backslash U)) \rightarrow(X, A ; S)$ induces an isomorphism $i_{*}: C H_{*}(X \backslash U, A \backslash U ; S \cap(X \backslash U)) \rightarrow(X, A ; S)$;

(vii) $C H_{*}(\mathrm{pt} ; \mathrm{pt})=H_{*}(\mathrm{pt})$.

Proof. (i), (ii) $\mathrm{CH}_{*}$ is a covariant functor, as it is a composition of covariant functors.

(iii), (iv) For every index pair $\left(N_{1}, N_{0}\right)$ for $S$ in $X$, the pair

$$
\left(N_{1} / N_{0},\left(N_{1} \cap A\right) /\left(N_{0} \cap A\right)\right)
$$

has a boundary operator $\partial$ which commutes with the induced maps of flow maps and makes the sequence

$$
\begin{gathered}
\cdots \stackrel{\partial}{\longrightarrow} H_{n}\left(\left(N_{1} \cap A\right) /\left(N_{0} \cap A\right), *\right) \stackrel{i_{*}}{\longrightarrow} H_{n}\left(N_{1} / N_{0}, *\right) \\
\stackrel{j_{*}}{\longrightarrow} H_{n}\left(N_{1} / N_{0},\left(N_{1} \cap A\right) /\left(N_{0} \cap A\right)\right) \stackrel{\partial}{\longrightarrow} \cdots
\end{gathered}
$$

exact. As this boundary operator is natural, it gives a well defined boundary operator on the inverse limits which satisfies (iii) and (iv).

(v) This is immediate from corollary 2.12 .

(vi) Let $\left(N, N_{0}\right)$ be an index pair for $S$ in $X$. $N_{0}$ is then a neighbourhood retract in $N$ via the flow, and $N_{0} \cap A$ is similarly a neighbourhood retract in $N \cap A$. It follows then that the quotient maps

$$
p_{1}:\left(N, N_{0} \cup(N \cap A)\right) \rightarrow\left(N / N_{0},(N \cap A) /\left(N_{0} \cap A\right)\right)
$$

and

$$
\begin{gathered}
p_{2}:\left(N \backslash U,\left(N_{0} \cup(N \cap A)\right) \backslash U\right) \rightarrow\left((N \backslash U) /\left(N_{0} \backslash U\right),\right. \\
\left.\left\{\left(N_{0} \cup(N \cap A)\right) \backslash U\right\} /\left(N_{0} \cap A \backslash U\right)\right)
\end{gathered}
$$

induce isomorphisms on homology. From the excision axiom of singular homology, the inclusion

$$
i_{*}: H_{*}\left(N \backslash U,\left(N_{0} \cup(N \cap A)\right) \backslash U\right) \rightarrow H_{*}\left(N, N_{0} \cup(N \cap A)\right)
$$

is an isomorphism. Hence

$$
\begin{aligned}
i_{*}: H_{*}\left((N \backslash U) /\left(N_{0} \backslash U\right),\left\{\left(N_{0} \cup(N \cap A)\right) \backslash U\right\} /\left(N_{0} \cap A \backslash U\right)\right) \\
\quad \rightarrow H_{*}\left(N / N_{0},(N \cap A) /\left(N_{0} \cap A\right)\right),
\end{aligned}
$$


which represents

$$
i_{*}: C H_{*}(X \backslash U, A \backslash U ; S \backslash U) \rightarrow C H_{*}(X, A ; S),
$$

is also an isomorphism.

(vii) This is the observation above that $\mathrm{CH}_{*}(S ; S)=H_{*}(S)$ for any compact metric space $S$.

The homotopy axiom is a special case of the following naturality statement for the homology Conley index under continuation:

THEOREM 3.7. If $X \times \Lambda$ is a parametrized family of flows, with $S_{\lambda} \subseteq X_{\lambda}$ and $S_{\mu} \subseteq X_{\mu}$ related by continuation, then $\mathrm{CH}_{*}\left(X_{\lambda} ; S_{\lambda}\right) \cong C H_{*}\left(X_{\mu}, S_{\mu}\right)$. Further, if $F: X \times \Lambda \rightarrow$ $Y \times \Lambda$ is a flow map, then there exists a commutative diagram

$$
\begin{array}{lrl}
C H_{*}\left(X_{\lambda} ; F_{\lambda}^{-1}\left(S_{\lambda}\right)\right) \underset{F_{\lambda^{*}}}{\longrightarrow} C H_{*}\left(Y_{\lambda}, S_{\lambda}\right) & \cong\left\lfloor F_{Y^{*}(\lambda, \mu)}\right. \\
\left.F_{X^{*}(\lambda, \mu)}\right\rfloor & \downarrow \\
C H_{*}\left(X_{\mu} ; F_{\mu}^{-1}\left(S_{\lambda}\right)\right) \underset{F_{\mu^{*}}}{\longrightarrow} C H_{*}\left(Y_{\mu}, S_{\mu}\right)
\end{array}
$$

We can equally well define a cohomology Conley index $\mathrm{CH}^{*}$ based on singular cohomology. As the morphisms in $I(X, A ; S)$ induce ring isomorphisms, this will give a cohomology theory on $\mathscr{H S}$ with cup product. Further, in different settings we can follow the Conley index functor $I$ by different (co-)homology functors $h$ and obtain corresponding Conley (co-)homology indices $C h$ (cf. [4]).

The exact sequence of a pair (theorem 3.6(iii)) can be used in a variety of ways to facilitate computations with the homology Conley index. In [10] a theorem on the structure of isolated invariant sets in a manifold $M$ is proved by showing that, if the theorem fails to hold, an invariant submanifold $M_{0}$ can be constructed so that the sequence of the pair $\left(M, M_{0} ; S\right)$ fails to be natural. A more positive application comes from the relation between chain recurrence and strongly gradientlike flows.

For example, if $X$ has compact maximal invariant set $S$, then the chain recurrent set $R$ of $X$ is compact. Form the quotient space $\tilde{X}$ by collapsing each component of $R$ to a point. Conley [1] shows that $\tilde{X}$ has a strongly gradient-like flow, and suggests that the flow on $X$ be studied by relating it to the flows on $R$ and $\tilde{X}$. In terms of the homology index, this is achieved by the exact sequence of $(X, R ; S)$. Namely, the natural projection $\pi: X \rightarrow \tilde{X}$ is a flow map and generates a commutative diagram

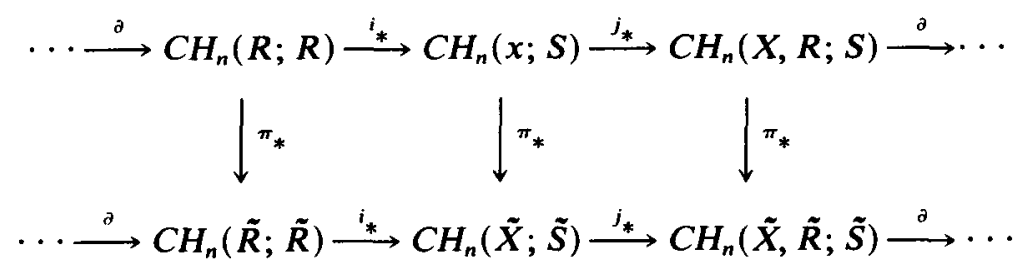

with $C H_{n}(R ; R)=H_{n}(R)$ and $C H_{n}(\tilde{R} ; \tilde{R})=H_{n}(\tilde{R})$. As $\tilde{R}$ consists of a finite set of points, $j_{*}: C H_{n}(\tilde{X} ; \tilde{S}) \rightarrow C H_{n}(\tilde{X}, \tilde{R} ; \tilde{S})$ is an isomorphism for $n>1$ and injective for $n=1$. Moreover, if $R$ is an equivariant neighbourhood retract in $X$, or if a 
continuous homology theory is used, then $\pi_{*}: C H_{n}(X, R ; S) \rightarrow C H_{n}(\tilde{X}, \tilde{R} ; \tilde{S})$ is an isomorphism. Then for $n \geq 1$ there is an exact sequence

$$
\cdots \rightarrow H_{n}(R) \stackrel{i_{*}}{\longrightarrow} C_{n}(X ; S) \stackrel{\pi_{*}}{\longrightarrow} C H_{n}(\tilde{X} ; \tilde{S}) \rightarrow \cdots
$$

relating the chain recurrent set and the homology indices in $X$ and $\tilde{X}$.

\section{Computational techniques for the homology Conley index}

Many of the basic computational methods of singular homology remain valid for the homology Conley index. As examples, we record analogues for the Conley index of the Mayer-Vietoris and Künneth theorems. As in singular theory, these theorems require the concept of an excisive triad, so we first consider excisiveness in the Conley index.

Proposition 4.1. Suppose $(X ; S) \in \mathscr{I S}$, with $X_{1}, X_{2}$ closed subflows of $X$. Let $X_{0}=X_{1} \cap X_{2}$ and let $S_{i}=X_{i} \cap S$. Then the following are equivalent:

(i) $i_{1^{*}}: C H_{*}\left(X_{1}, X_{0} ; S_{1}\right) \rightarrow C H_{*}\left(X, X_{2} ; S\right)$ is an isomorphism;

(ii) $i_{2^{*}}: \mathrm{CH}_{*}\left(X_{2}, X_{0} ; S_{2}\right) \rightarrow C H_{*}\left(X, X_{1} ; S\right)$ is an isomorphism;

(iii) $\left(i_{1^{*}}, i_{2^{*}}\right): C H_{*}\left(X_{1}, X_{0} ; S_{1}\right) \oplus C H_{*}\left(X_{2}, X_{0} ; S_{2}\right) \rightarrow C H_{*}\left(X, X_{0} ; S\right)$ is an isomorphism.

Proof. Choose a regular index pair $\left(N_{1}, N_{0}\right)$ for $S$ in $X$, and let $N_{i j}=N_{i} \cap X_{j}$. We then have exact sequences of chain complexes

$$
\begin{aligned}
0 & \rightarrow \frac{S_{*}\left(N_{11}\right)}{S_{*}\left(N_{01}\right)+S_{*}\left(N_{10}\right)} \stackrel{i_{1}}{\longrightarrow} \frac{S_{*}\left(N_{1}\right)}{S_{*}\left(N_{0}\right)+S_{*}\left(N_{12}\right)} \rightarrow \frac{S_{*}\left(N_{1}\right)}{S_{*}\left(N_{11}\right)+S_{*}\left(N_{0}\right)+S_{*}\left(N_{12}\right)} \rightarrow 0, \\
0 & \rightarrow \frac{S_{*}\left(N_{12}\right)}{S_{*}\left(N_{02}\right)+S_{*}\left(N_{10}\right)} \stackrel{i_{2}}{\longrightarrow} \frac{S_{*}\left(N_{1}\right)}{S_{*}\left(N_{0}\right)+S_{*}\left(N_{11}\right)} \rightarrow \frac{S_{*}\left(N_{1}\right)}{S_{*}\left(N_{11}\right)+S_{*}\left(N_{0}\right)+S_{*}\left(N_{12}\right)} \rightarrow 0, \\
0 & \rightarrow \frac{S_{*}\left(N_{11}\right)+S_{*}\left(N_{12}\right)}{S_{*}\left(N_{01}\right)+S_{*}\left(N_{10}\right)+S_{*}\left(N_{02}\right)} \stackrel{\left(i_{1}, i_{2}\right)}{\longrightarrow} \frac{S_{*}\left(N_{1}\right)}{S_{*}\left(N_{10}\right)+S_{*}\left(N_{0}\right)} \\
& \rightarrow \frac{S_{*}\left(N_{1}\right)}{S_{*}\left(N_{11}\right)+S_{*}\left(N_{0}\right)+S_{*}\left(N_{12}\right)} \rightarrow 0 .
\end{aligned}
$$

(i)-(iii) hold if and only if $i_{1}, i_{2}$ and $\left(i_{1}, i_{2}\right)$ are chain equivalences, hence if and only if $H_{*}\left(S_{*}\left(N_{1}\right) / S_{*}\left(N_{11}\right)+S_{*}\left(N_{0}\right)+S_{*}\left(N_{12}\right)\right)=0$. Thus they are all equivalent.

Definition 4.2. A triad $\left\{(X, A ; S) ;\left(X_{1}, A_{1} ; S_{1}\right),\left(X_{2}, A_{2} ; S_{2}\right)\right\}$ with $A_{i} \subseteq A \cap X_{i}$ is excisive if the inclusions $i_{1^{*}}: C H_{*}\left(X_{1}, X_{0} ; S_{1}\right) \rightarrow C H_{*}\left(X, X_{2} ; S\right)$ and $i_{1^{*}}: C H_{*}\left(A_{1}, A_{0} ; S \cap A_{1}\right) \rightarrow C H_{*}\left(A, A_{2} ; S \cap A\right)$ are isomorphisms.

Proposition 4.3. If $X_{1}, X_{2} \subseteq X$ are closed subflows whose interiors cover $X$ and $A_{i}=A \cap Y_{i}$, then for every $(X, A ; S) \in \mathscr{I S}$ the triad

$$
\left\{(X, A ; S) ;\left(X_{1}, A_{1} ; S_{1}\right),\left(X_{2}, A_{2} ; S_{2}\right)\right\}
$$

is excisive.

Proof. Let $N_{i j}$ be as in proposition 4.1. Then $N_{11}$ and $N_{12}$ are closed subsets of $N_{1}$ whose interiors cover $N_{1}$, so $S_{*}\left(N_{1}\right) \simeq S_{*}\left(N_{11}\right)+S_{*}\left(N_{12}\right)$. In particular, 
$H_{*}\left(S_{*}\left(N_{1}\right) / S_{*}\left(N_{11}\right)+S_{*}\left(N_{0}\right)+S_{*}\left(N_{12}\right)\right)=0$. Similarly, the interiors of $A \cap N_{11}$ and $A \cap N_{12}$ cover $A \cap N_{1}$.

Proposition 4.4. If $X_{1}, X_{2} \subseteq X$ are closed subflows so that $X=X_{1} \vee X_{2}, A_{i}=A \cap X_{i}$ and $X_{0}$ is a neighbourhood retract in $X$, then for every $(X, A ; S) \in \mathscr{P},\{(X, A ; S)$; $\left.\left(X_{1}, A_{1} ; S_{1}\right),\left(X_{2}, A_{2} ; S_{2}\right)\right\}$ is excisive.

Proof. Choose a regular index pair $\left(N_{1}, N_{0}\right)$ for $S$ in $X$. Then $C H_{*}\left(X_{1}, X_{0} ; S_{1}\right)$ and $C H_{*}\left(X, X_{2} ; S\right)$ are represented by $H_{*}\left(N_{11}, N_{01} \cup N_{10}\right)$ and $H_{*}\left(N_{1}, N_{0} \cup N_{12}\right)$. As $X_{0}$ is a neighbourhood retract, we can excise $N_{12} \backslash N_{10}$ from $N_{0} \cup N_{12}$, and $i_{1^{*}}: C H_{*}\left(X_{1}, X_{0} ; S_{1}\right) \rightarrow C H_{*}\left(X, X_{2} ; S\right)$ is an isomorphism.

TheOREM 4.5 (Mayer-Vietoris theorem for the Conley index). Let $(X, A ; S) \in \mathscr{I} \mathscr{S}$. For $i=1,2$, suppose $\left(X_{i}, A_{i} ; S_{i}\right) \subseteq(X, A ; S)$ with $X_{i}$ a closed subflow of $X, S_{i}=X_{i} \cap S$, and $\left\{(X, A ; S) ; \quad\left(X_{1}, A_{1} ; S_{1}\right), \quad\left(X_{2}, A_{2} ; S_{2}\right)\right\}$ excisive. Let $\left(X_{0}, A_{0} ; S_{0}\right)=$ $\left(X_{1} \cap X_{2}, A_{1} \cap A_{2} ; S_{1} \cap S_{2}\right)$. Then there exists a natural exact sequence

$$
\begin{aligned}
\cdots & \rightarrow C H_{*}\left(X_{0}, A_{0} ; S_{0}\right) \rightarrow C H_{*}\left(X_{1}, A_{1} ; S_{1}\right) \oplus C H^{*}\left(X_{2}, A_{2} ; S_{2}\right) \\
& \rightarrow C H_{*}(X, A ; S) \rightarrow \cdots .
\end{aligned}
$$

Proof. Choose a regular index pair for $S$ in $X$. Then the following sequences of chain complexes are exact:

$$
\begin{gathered}
0 \rightarrow \frac{S_{*}\left(N_{10} \cap A\right)}{S_{*}\left(N_{00} \cap A\right)} \rightarrow \frac{S_{*}\left(N_{11} \cap A\right)}{S_{*}\left(N_{01} \cap A\right)} \oplus \frac{S_{*}\left(N_{12} \cap A\right)}{S_{*}\left(N_{02} \cap A\right)} \rightarrow \frac{S_{*}\left(N_{11} \cap A\right)+S_{*}\left(N_{12} \cap A\right)}{S_{*}\left(N_{01} \cap A\right)+S_{*}\left(N_{02} \cap A\right)} \rightarrow 0, \\
0 \rightarrow \frac{S_{*}\left(N_{10}\right)}{S_{*}\left(N_{00}\right)} \rightarrow \frac{S_{*}\left(N_{11}\right)}{S_{*}\left(N_{01}\right)} \oplus \frac{S_{*}\left(N_{12}\right)}{S_{*}\left(N_{02}\right)} \rightarrow \frac{S_{*}\left(N_{11}\right)+S_{*}\left(N_{12}\right)}{S_{*}\left(N_{01}\right)+S_{*}\left(N_{02}\right)} \rightarrow 0 .
\end{gathered}
$$

As was noted in proposition 4.1, the hypothesis $\mathrm{CH}_{*}\left(X_{1}, X_{0} ; S_{1}\right) \cong \mathrm{CH}_{*}\left(X, X_{2} ; S\right)$ is equivalent to

$$
\frac{S_{*}\left(N_{1}\right)}{S_{*}\left(N_{11}\right)+S_{*}\left(N_{0}\right)+S_{*}\left(N_{12}\right)} \simeq 0,
$$

which is in turn equivalent to

$$
\frac{S_{*}\left(N_{11}\right)+S_{*}\left(N_{12}\right)}{S_{*}\left(N_{01}\right)+S_{*}\left(N_{02}\right)}=\frac{S_{*}\left(N_{1}\right)}{S_{*}\left(N_{0}\right)} .
$$

That is, (4.7) generates the long exact sequence

$$
\cdots \rightarrow C H_{*}\left(X_{0} ; S_{0}\right) \rightarrow C H_{*}\left(X_{1} ; S_{1}\right) \oplus C H_{*}\left(X_{2} ; S_{2}\right) \rightarrow C H_{*}(X ; S) \rightarrow \cdots .
$$

Similarly, (4.6) generates the long exact sequence

$$
\begin{aligned}
\cdots & \rightarrow C H_{*}\left(A_{0} ; A_{0} \cap S_{0}\right) \rightarrow C H_{*}\left(A_{1} ; A_{1} \cap S_{1}\right) \oplus C H_{*}\left(A_{2} ; A_{2} \cap S_{2}\right) \\
& \rightarrow C H_{*}(A ; A \cap S) \rightarrow \cdots .
\end{aligned}
$$

The quotient of (4.7) by (4.6) then generates the desired sequence.

Example 4.8. Consider the flow on the torus $\mathscr{Y}_{0}(\theta, \phi)=(\sin (\theta) \cos (\phi), \sin (\phi))$ given in 2.13. The set $S=(\phi=0, \pi)$ is isolated. We compute its index in $T^{2}$ from the Mayer-Vietoris sequence. Take $X_{1}=\{-\pi \leq \phi \leq 0\}, X_{2}=\{0 \leq \phi \leq \pi\}$. The homeomorphism $\phi \mapsto-\phi$ is a flow map which takes $S$ to $S$ and $X_{1}$ to $X_{2}$, so $C H_{*}\left(X_{1} ; S\right) \cong$ 
$C H_{*}\left(X_{2} ; S\right), C H_{*}\left(X_{1}, X_{0} ; S\right) \cong C H_{*}\left(X_{2}, X_{0} ; S\right)$, etc. An index pair for $S$ in $T^{2}$ is $N_{1}=\{-\pi \leq \phi \leq \varepsilon-\pi,-\varepsilon \leq \phi \leq \varepsilon, \pi-\varepsilon \leq \phi \leq \pi\}, \quad N_{0}=\{\phi=-\varepsilon, \varepsilon\}$. By excision, $H_{*}\left(N_{11}, N_{01} \cup N_{10}\right) \cong H_{*}\left(N_{1}, N_{0} \cup N_{12}\right)$ (i.e. $C H_{*}\left(X_{1}, X_{0} ; S\right) \cong C H_{*}\left(X, X_{2} ; S\right)$ ) so $\left\{(X ; S) ;\left(X_{1} ; S\right),\left(X_{2} ; S\right)\right\}$ is excisive, and there is an exact sequence

$$
\cdots \rightarrow C H_{n}\left(X_{0} ; S\right) \rightarrow C H_{n}\left(X_{1} ; S\right) \oplus C H_{n}\left(X_{2} ; S\right) \rightarrow C H_{n}(X ; S) \rightarrow \cdots .
$$

As $\quad S=X_{0}, \quad C H_{*}\left(X_{0} ; S\right)=H_{*}(S)=(\mathbb{Z} \oplus \mathbb{Z}, \mathbb{Z} \oplus \mathbb{Z}, 0,0, \ldots), \quad C H_{*}\left(X_{1} ; S\right) \cong$ $C H_{*}\left(X_{2} ; S\right) \cong(\mathbb{Z}, \mathbb{Z}, 0,0, \ldots)$, with $i_{*}: C H_{n}\left(X_{0} ; S\right) \rightarrow C H_{n}\left(X_{1} ; S\right)$ the projection onto a factor for $n=0,1$. From this, the sequence computes $\mathrm{CH}_{*}(X ; S) \cong$ $(\mathbb{Z}, \mathbb{Z} \oplus \mathbb{Z}, \mathbb{Z}, 0,0, \ldots)$.

TheOREM 4.9 (Künneth theorem for the Conley index). If $(X, A ; S),(Y, B ; T) \in \mathscr{I} \mathscr{S}$ so that $\{(X \times B \cup A \times Y, \varnothing ; S \times(B \cap T) \cup(S \cap A) \times T) ;(A \times Y, \varnothing ;(S \cap A) \times T)$, $(X \times B, \varnothing ; S \times(B \cap T))\}$ is an excessive triad, then there exists a natural exact sequence

$$
\begin{aligned}
0 & \rightarrow C H_{*}(X, A ; S) \otimes C H_{*}(Y, B ; T) \\
& \rightarrow C H_{*}(X \times Y, X \times B \cup A \times Y ; S \times(B \cap T) \cup(S \cap A) \times T) \\
& \rightarrow C H_{*}(X, A ; S) * C H_{*}(Y, B ; T) \rightarrow 0
\end{aligned}
$$

which is split exact.

Proof. Choose regular index pairs $\left(M_{1}, M_{0}\right)$ for $S$ in $X$ and $\left(N_{1}, N_{0}\right)$ for $T$ in $Y$. Then $\left(M_{1}, M_{0}\right) \times\left(N_{1}, N_{0}\right)$ is regular index pair for $S \times T$ in $X \times Y$. Let $A_{i}=A \cap M_{i}$, $B_{i}=B \cap N_{i}$. Then $C H_{*}(X, A ; S)$ and $C H_{*}(Y, B ; T)$ are represented by $H_{*}\left(M_{1}, M_{0} \cup A_{1}\right)$ and $H_{*}\left(N_{1}, N_{0} \cup B_{1}\right)$, and

$$
C H_{*}(X \times Y,(X \times B) \cup(A \times Y) ; S \times(B \cap T) \cup(S \cap A) \times T)
$$

is represented by $H_{*}\left(M_{1} \times N_{1},\left(M_{0} \cup A_{1}\right) \times N_{1} \cup M_{1} \times\left(N_{0} \cup B_{1}\right)\right)([1]$, Ch. III, $\S 6)$. The excision hypothesis allows us to apply the Künneth theorem of singular theory to $\left(M_{1}, M_{0} \cup A_{1}\right) \times\left(N_{1}, N_{0} \cup B_{1}\right)$, which gives the result.

As the sequences in theorems 4.5 and 4.9 are natural, they remain exact under continuation (in 4.5 we must require that the sets $X_{i}$ and $A_{i}$ remain invariant throughout the continuation), and the theorems are 'stable' under continuation.

\section{Lefschetz theorem for the Conley index}

We now develop an analogue of the Lefschetz fixed-point theorem. Given a self-map $f$ on a flow $X$ with $f(S) \subseteq S$ (and some technical hypotheses), $C H_{*}(X ; S)$ to compute a Lefschetz number in place of $H_{*}(S)$. Similar to the ordinary Lefschetz number, this will detect fixed points of flow maps (theorem 5.7). Such a fixed-point theorem will have (at least) two uses. First, many problems in differential equations can be translated into searches for fixed points of maps. If this translation leads to a search for fixed points of a flow map on an isolated invariant set $S$, this result will be applying the classical Lefschetz theorem to $f$ or to $f \mid S$. In particular, the ConleyLefschetz number will not depend on the topology of $S$, so this result may be able to detect fixed points when the classical Lefschetz number $L(f \mid S)$ is not defined, not computable, or zero. Second, knowing that $S \cap$ Fix $(f)$ is non-empty may give 
further information about $S$. For example, if a group acts on $X$, the fixed-point set of multiplication by a group element may be computable, If $S$ can be shown to have fixed points under this multiplication, then $S$ has been (partially) located.

To develop the theorem, we require an appropriate class of self-maps on flows and some ANR hypotheses on index pairs.

Definition 5.1. Let $Y$ be a locally compact metric flow, $(Y, X ; S) \in \mathscr{I S}$ with $S \subseteq$ int ${ }_{Y} X$. A morphism $f:\left(X ; f^{-1}(S)\right) \rightarrow(Y ; S)$ is a self-map on $(X ; S)$ if $f(S) \subseteq S$ and $S$ is an attractor in $f^{-1}(S)$.

That is, $f$ is a self-map on $S$ and is defined on an invariant neighbourhood of $S$. With the attractor condition, $S$ has a dual repeller in $f^{-1}(S)$, which we will denote by $S^{*}$. The atractor-repeller pair $\left(f^{-1}(S) ; S, S^{*}\right)$ has associated to it an index triple in $X$ : a compact triple $\left(\tilde{N}_{2}, \tilde{N}_{1}, \tilde{N}_{0}\right)$ with $\left(\tilde{N}_{2}, \tilde{N}_{0}\right)$ an index pair for $f^{-1}(S) ;\left(\tilde{N}_{1}, \tilde{N}_{0}\right)$ an index pair for $S$; and $\left(\tilde{N}_{2}, \tilde{N}_{1}\right)$ an index pair for $S^{*}$ (all relative to $X$ ).

If $\left(N, N_{0}\right)$ is an index pair for $S$ in $X$, then it is an index pair for $S$ in $Y$ as well, and $\left(f^{-1}(N), f^{-1}\left(N_{0}\right)\right)$ is an index pair for $f^{-1}(S)$ in $X$. In [8] Kurland shows that a compact $N^{\prime} \subseteq f^{-1}(N)$ can be chosen so that $\left(f^{-1}(N), N^{\prime}, f^{-1}\left(N_{0}\right)\right)$ is an index triple for $\left(f^{-1}(S) ; S, S^{*}\right)$. As both $\left(N, N_{0}\right)$ and $\left(N^{\prime}, f^{-1}\left(N_{0}\right)\right)$ are index pairs for $S$, the map $\varphi^{\prime}: N / N_{0} \rightarrow N^{\prime} / g^{-1}\left(N_{0}\right)$ is a homotopy equivalence for $t$ sufficiently large. The composition

$$
N / N_{0} \stackrel{\varphi^{\prime}}{\longrightarrow} N^{\prime} / f^{-1}\left(N_{0}\right) \stackrel{i}{\longrightarrow} f^{-1}(N) / f^{-1}\left(N_{0}\right) \stackrel{\vec{f}}{\longrightarrow} N / N_{0}
$$

then defines the induced homology map $f_{*}: \mathrm{CH}_{*}(X ; S) \rightarrow \mathrm{CH}_{*}(Y ; S)$. Since $S \subseteq$ int $_{Y} X, i_{*}: C H_{*}(X, S) \rightarrow C H_{*}(Y ; S)$ is an isomorphism (induced by the identity on $\left.N / N_{0}\right)$, and $f$ induces a homology self-map $i_{*}^{-1} \circ f_{*}$ on $C_{*}(X ; S)$. All homology groups will now be taken with rational coefficients.

Definition 5.2. If $(X ; S) \in \mathscr{I S}$ and $f$ is a self-map on $(X ; S)$, the Conley-Lefschetz number of $f$ is $L(f ; X, S) \equiv \sum_{n=0}^{\infty}(-1)^{n} \operatorname{tr}\left(i_{n^{*}}^{-1} \circ f_{n^{*}}\right)$. The Conley-Euler number of $(X ; S)$ is $\chi(X ; S)=L(\mathrm{id}, X ; S)$.

The class of maps chosen in 5.1 is essentially the largest for which $L(f ; X, S)$ can be defined in terms of self-maps on index pairs, and is singled out for that reason. Note that when $\mathrm{CH}_{*}(X ; S)$ is finitely generated (so that the sum above is finite), $L(f, X ; S)$ is well defined. For, if $\left(N, N_{0}\right)$ and $\left(\tilde{N}, \tilde{N}_{0}\right)$ are index pairs for $S$ in $X$, the diagram

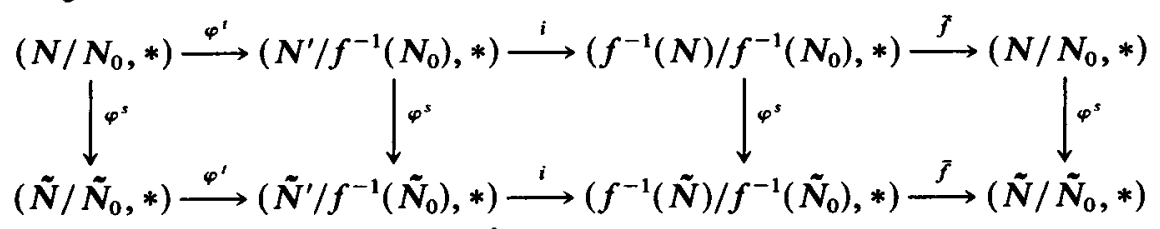

commutes for sufficiently large $s$ and $t$. The two horizontal compositions are then identified after applying $H_{*}$ taking the appropriate inverse limits, $\operatorname{so} \operatorname{tr}\left(i_{n^{*}}^{-1} \circ f_{n^{*}}\right)$ is independent of the choice of index pair.

To find when $L(f ; X, S)$ is defined, and to relate it to fixed points of $f$, we consider the classes of index pairs $\mathcal{N}(X ; S)$ and $\mathscr{A N}(X ; S)$. 
LeMmA 5.3. If $\left(N, N_{0}\right) \in \mathcal{N}(X ; S)$, let $N^{T}=\{x \in N \mid x \cdot[-T, T] \subseteq N\}, \quad N_{0}^{T}=$ $N_{0} \cdot(-T) \cap N^{T}$. Then $\left(N^{T}, N_{0}^{T}\right) \in \mathcal{N}(X ; S)$ for every $t \geq 0$.

Proof. $\left(N^{T}, N_{0}^{T}\right)$ is clearly a compact pair.

(i) $S \subseteq \operatorname{cl}_{X}\left(N^{T} \backslash N_{0}^{T}\right) \subseteq \operatorname{cl}_{X}\left(N \backslash N_{0}\right)$, so $S=I\left(\operatorname{cl}_{X}\left(N^{T} \backslash N_{0}^{T}\right)\right)$. If $S \not \subset$ int $_{X}\left(N^{T} \backslash N_{0}^{T}\right)$, then there exists a sequence $\left\{x_{n}\right\}$ converging to $x \in S$ with $x_{n} \notin$ $N^{T} \backslash N_{0}^{T}$, so with $x_{n} \cdot[-T, T] \not \subset N \backslash N_{0}$. Choose $t_{n} \in[-T, T]$ so that $x_{n} \cdot t_{n} \notin N \backslash N_{0}$. Then (taking a subsequence if necessary) $t_{n} \rightarrow t \in[-T, T]$ and $x_{n} \cdot t_{n} \rightarrow x \cdot t$. But $x \in S$, so $x \cdot t \in S \subseteq$ int $_{X}\left(N \backslash N_{0}\right)$ and $\left\{x_{n} \cdot t_{n}\right\} \subseteq X \backslash\left(N \backslash N_{0}\right)$ is bounded away from $x \cdot t$. This is a contradiction, so $S \subseteq \operatorname{int}_{X}\left(N^{T} \backslash N_{0}^{T}\right)$.

(ii) If $x \in N_{0}^{T}$ and $x \cdot[0, t] \subseteq N^{T}$, then $x \cdot T \in N_{0}$ and $x \cdot[-T, t+T] \subseteq N$. In particular, $x \cdot T \in N_{0}$ and $(x \cdot T) \cdot[0, t] \subseteq N$, so $(x \cdot T) \cdot[0, t] \subseteq N_{0}$. That is, $x \cdot[0, t] \subseteq$ $N_{0} \cdot(-T) \cap N^{T}=N_{0}^{T}$.

(iii) If $x \in N^{T}, x \cdot \mathbb{R}^{+} \not \subset N^{T}$, then $x \cdot \mathbb{R}^{+} \not \subset N$. There exists then a $t \geq 0$ so that $x \cdot[0, t] \subseteq N, x \cdot t \in N_{0}$. Thus $x \cdot[-T, t] \subseteq N, x \cdot t \in N_{0}$. If $t \geq T$, then $x \cdot[0, t-T] \subseteq$ $N$ and $x \cdot(t-T) \in N_{0} \cdot(-T)$, so $x \cdot(t-T) \subseteq N_{0}^{T}$. If $t \leq T$, then $x \cdot T \in N_{0}$.

(iv) Let $\tau_{+T}, \tau_{-T}: N^{T} \rightarrow \mathbb{R}$ be the exit-time and entrance-time maps for $\left(N^{T}, N_{0}^{T}\right)$. Then $\tau_{+T}(x)=\tau_{+0}(x)-T$ and $\tau_{-T}(x)=\tau_{-0}(x)+T$.

If $x \in N^{T}$, then $T \leq \tau_{+0}(x)$. If $\tau_{+0}(x)=T$, then $x \in N_{0}^{T}$ and $\tau_{+T}(x)=0$. If $x \in N^{T}$ with $T<\tau_{+0}(x)<\infty$, then $x \in N^{T} \backslash N_{0}^{T} \cdot x \cdot \tau_{+0}(x) \in N_{0}$, so

$$
x \cdot\left(\tau_{+0}(x)-T\right) \in N_{0} \cdot(-T) \text { and } \tau_{+T}(x) \leq \tau_{+0}(x)-T .
$$

If $\tau_{+T}(x)<\tau_{+0}(x)-T$, then $\tau_{+0}(x)>\tau_{+T}(x)-T$ and $x \cdot\left[0, \tau_{+T}(x)-T\right] \subseteq N \backslash N_{0}$. But $x \cdot \tau_{+T}(x) \in N_{0}^{T} \subseteq N_{0} \cdot(-T)$, so $x \cdot\left(\tau_{+T}(x)-T\right) \in N_{0}$. This is a contradiction, so $\tau_{+T}(x)=\tau_{+0}(x)-T$. If $\tau_{+0}(x)=\infty$, then $x \cdot \mathbb{R}^{+} \subseteq N \backslash N_{0}$. If $x \in N^{T}, t>0$ so that $x \cdot t \in N_{0}^{T}$, then $x \cdot(t+T) \in N_{0}$. There is no such $t$, so $x \cdot \mathbb{R}^{+} \subseteq N^{T} \backslash N_{0}^{T}$ and $\tau_{+T}(x)=$ $\infty$. This gives the equality for $\tau_{+T}$. The equality for $\tau_{-T}$ is analogous. And if $x \cdot \tau_{+0}(x)$ leaves $N$ immediately, then $x \cdot\left(\tau_{+0}(x)-T\right)$ leaves $N^{T}$ immediately, so $\tau_{+T}(x)=$ $\sup \left\{t \geq 0 \mid x \cdot[0, t] \subseteq N^{\top}\right\}$.

LeMma 5.4. If $\left(N, N_{0}\right) \in \mathscr{A} \mathcal{N}(X ; S)$, then $\left(N^{T}, N_{0}^{T}\right) \in \mathscr{A} \mathcal{N}(X ; S)$ for every $T \geq 0$.

Proof. It suffices to show that there exist maps $r_{T}: N / N_{0} \rightleftarrows N^{T} / N_{0}^{T}: i_{T}$ so that $r_{T} \circ i_{T}=$ id.

Let $i_{T}([x])=[x \cdot T]$. As $N_{0}^{T} \cdot T \subseteq N_{0}$, this is well defined and continuous on $N^{T} / N_{0}^{T}$.

Let

$$
r_{T}[x]= \begin{cases}{[x \cdot(-T)]} & \text { if }-\tau_{-0}(x) \geq 2 T, \\ {\left[x \cdot\left(\tau_{-0}(x)+T\right]\right.} & \text { if }-\tau_{-0}(x) \leq 2 T \leq \tau_{+0}(x)-\tau_{-0}(x), \\ {\left[N_{0}^{T}\right]} & \text { if } \tau_{+0}(x)-\tau_{-0}(x) \leq 2 T .\end{cases}
$$

The definitions agree on the overlaps and take all of $N_{0}$ to $N_{0}^{T}$, so $r_{T}$ is also well defined and continuous. If $x \in N^{T}$, then $-\tau_{-0}(x) \geq T$, so $-\tau_{-0}(x \cdot T) \geq 2 T$ and $r_{T} \circ i_{T}[x]=[(x \cdot T) \cdot(-T)]=[x]$.

Definition 5.5. Suppose $f$ is a self-map on $(X ; S) \in \mathscr{S} \mathscr{S}$. For every $\left(N, N_{0}\right) \in$ $\mathscr{A N}(X ; S)$, choose an $N^{\prime}$ so that $\left(f^{-1}(N), N^{\prime}, f^{-1}\left(N_{0}\right)\right)$ is an index triple for $\left(f^{-1}(S) ; S, S^{*}\right)$. Let

$$
T\left(f ; N, N_{0}\right)=\inf \left\{T>0 \mid\left(N \backslash N_{0}\right)^{T} \subseteq N^{\prime} \backslash f^{-1}\left(N_{0}\right),\left(N^{\prime} \backslash f^{-1}\left(N_{0}\right)\right)^{T} \subseteq N \backslash N_{0}\right\}
$$


and let

$$
T(f ; X, S)=\inf \left\{T\left(f ; N, N_{0}\right) \mid\left(N, N_{0}\right) \in \mathscr{A} \mathcal{N}(X ; S)\right\} .
$$

That is, $T(f ; X, S)$ is the minimum time needed in the definition of $L(f ; X, S)$. An example below shows that $T(f ; X, S)>0$ in general .

LeMmA 5.6. If $\left(N, N_{0}\right) \in \mathscr{A N}(X ; S)$ and $\left(f^{-1}(N), N^{\prime}, f^{-1}\left(N_{0}\right)\right)$ is an index triple for $\left(f^{-1}(S) ; S, S^{*}\right)$, let $N^{\prime}(T)=N^{\prime T} \cup f^{-1}\left(N_{0}^{T}\right)$. Then $\left(f^{-1}\left(N^{T}\right), N^{\prime}(T), f^{-1}\left(N_{0}^{T}\right)\right)$ is an index triple for $\left(f^{-1}(S) ; S, S^{*}\right)$ and $T\left(f ; N^{T}, N_{0}^{T}\right)<T\left(f ; N, N_{0}\right)$ for every $T \geq 0$. Proof. (i) To show $\left(f^{-1}\left(N^{T}\right), N^{\prime}(T), f^{-1}\left(N_{0}^{T}\right)\right)$ is an index triple, it suffices to show $\left(N^{\prime}(T), f^{-1}\left(N_{0}^{T}\right)\right)$ is an index pair for $S$. But $\left(N^{\prime T}, f^{-1}\left(N_{0}\right) \cdot(-T) \cap\left(N^{\prime T}\right)\right.$ is an index pair for $S$ by lemma 5.3, and $\left(N^{\prime}(T), f^{-1}\left(N_{0}^{T}\right)\right)$ adds a positively invariant set to the exit set, so it too is an index pair.

(ii) $N^{\prime}(T) \backslash f^{-1}\left(N_{0}^{T}\right)=N^{\prime T} \backslash f^{-1}\left(N_{0}\right) \cdot(-T) \cap N^{\prime T}=\left(N^{\prime} \backslash f^{-1}\left(N_{0}\right)\right)^{T}$. The lemma then follows from the observations that $\left(A^{t}\right)^{s}=A^{t+s}$ for every $t, s>0$ and that $A^{\prime} \subseteq B^{\prime}$ if $A \subseteq B$.

Theorem 5.7 (Lefschetz theorem for the Conley index). If $f$ is a self-map on $(X ; S) \in \mathscr{I S}$ and $\mathscr{A N}(X ; S)$ is non-empty, then $L(f ; X, S)$ is defined. Further, if $L(f ; X, S) \neq 0$, then for every $g$ homotopic to $f$ as flow maps and every $t \geq T(g ; X, S)$, the composition $g \circ \varphi_{3 t}$ has fixed points in $S$.

Proof. (i) If $\left(N, N_{0}\right) \in \mathscr{A} \mathcal{N}(X ; S)$, then $C H_{*}(X ; S)=H_{*}\left(N / N_{0}, *\right)$ is the reduced homology of a compact ANR. It is then finitely generated, so $L(f ; X, S)$ is given by a finite sum.

(ii) First consider $g=f$. Choose a $t>T(f ; X, S)$. There exists an $\left(N, N_{0}\right) \in$ $\mathscr{A} \mathcal{N}(X ; S)$ with $T\left(f ; N, N_{0}\right)<t$. For every $T \geq 0$, let $U^{T}=\left\{x \in N^{T} \mid \tau_{+T}(x) \leq t\right\}$. Then $\varphi_{T}^{\prime}: N^{T} / N_{0}^{T} \rightarrow N^{\prime}(T) / f^{-1}\left(N_{0}^{T}\right)$ is continuous (as $t>T\left(f ; N^{T}, N_{0}^{T}\right)$ for every $T$ ) and has $\varphi_{T}^{t}\left(U^{T} / N_{0}^{T}\right)=\left[f^{-1}\left(N_{0}^{T}\right)\right]$. That is, $\varphi_{T}^{t}([x]) \neq\left[f^{-1}\left(N_{0}^{T}\right)\right]$ only if $x \cdot[0,2 t] \subseteq$ $N^{T} \backslash N_{0}^{T}$, but every $x \in U^{T}$ has $\tau_{+0}(x) \in[0,2 T]$ and $x \cdot \tau_{+0}(x) \in N_{0}^{T}$. Further, $U^{T}$ is compact, deformable along orbits into $N_{0}^{T}$, with $U^{T} / N_{0}^{T}$ an ANR (as it is a neighbourhood retract in the ANR $N^{T} / N_{0}^{T}$ ). We then have the commutative diagram

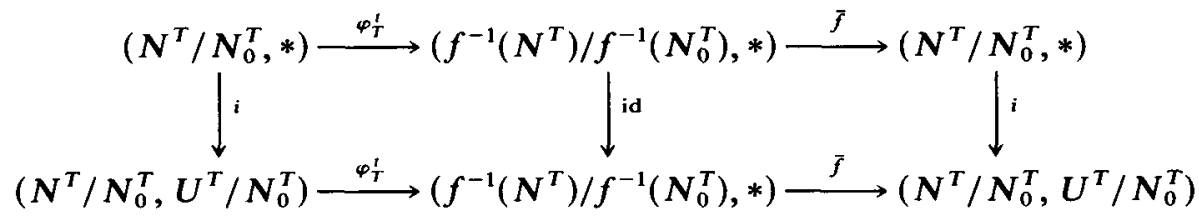

with the inclusion $i:\left(N^{T} / N_{0}^{T}, *\right) \rightarrow\left(N^{T} / N_{0}, U^{T} / N_{0}^{T}\right)$ an isomorphism on homology. In particular, $\bar{f} \circ \varphi_{T}^{i}:\left(N^{T} / N_{0}, U^{T} / N_{0}^{T}\right) \rightarrow\left(N^{T} / N_{0}, U^{T} / N_{0}^{T}\right)$ has $L\left(\bar{f} \circ \varphi_{T}^{t}\right)=L(f ; X, S)$. Thus $\left(N^{T} / N_{0}, U^{T} / N_{0}^{T}\right)$ is a compact ANR pair with $L\left(\bar{f} \circ \varphi_{T}^{t}\right) \neq 0$, so $\bar{f} \circ \varphi_{T}^{\prime}$ has fixed points in $\operatorname{cl}_{X}\left(N^{T} \backslash U^{T}\right)$ by the Lefschetz theorem for compact ANR pairs.

As this holds for every $T$, it holds for $S=\bigcap\left\{\operatorname{cl}_{X}\left(N^{T} \backslash U^{T}\right) \mid T \geq 0\right\}$, so $\bar{f} \circ \varphi_{T}^{t}$ has fixed points in $S$. But on $S, \bar{f} \circ \varphi_{T}^{t}=f \circ \varphi_{3 t}$, so $f \circ \varphi_{3 t}$ has fixed points in $S$. This is true for every $t>T(f ; X, S)$, so by continuity it is also true for $t=T(f ; X, S)$.

(iii) If $F: f=g$ is a homotopy of flow maps, then $f^{-1}(S)=g^{-1}(S)$, so $S$ is an attractor in $g^{-1}(S)$. As $f_{*}=g_{*}, L(f ; X, S)=L(g ; X, S)$. The hypothesis that 
$\mathscr{A} \mathcal{N}(X, S)$ is non-empty is independent of the flow map being considered, so we may apply (i) to $g$.

Corollary 5.8. If $(X ; S) \in \mathscr{I S}$ such that $\mathscr{A N}(X ; S)$ is non-empty, then $\chi(X ; S)$ is defined. If $\chi(X ; S) \neq 0$, then $S$ contains a fixed point of the flow.

Proof. Take $X=Y$ and $f=$ id. Then $S$ is an attractor in $f^{-1}(S)=S$ and $T(g ; X, S)=0$. Then for every $t \geq 0$, Fix $\left(\left.\varphi_{1}\right|_{s}\right) \neq \varnothing$. If $S$ does not contain a fixed point of the flow, then it contains periodic points of arbitrarily small period. Take a convergent sequence of such points, with periods going to zero. Take a limit $x_{0} \in S$. Then for every neighbourhood $U$ of $x_{0}$ and every $t \in \mathbb{R}, U \cap(U \cdot t) \neq \varnothing$.

If $x_{0}$ is not a rest point, then there exists a $t>0$ such that $x_{0} \neq x_{0} \cdot t$. Choose disjoint open sets $U_{1}, U_{2}$ around $x_{0}$ and $x_{0} \cdot t$. Let $V_{1}=U_{1} \cap\left(U_{2} \cdot(-t)\right)$ and let $V_{2}=V_{1} \cdot t$. Then $x_{0} \in V_{1}$ with $V_{i} \subseteq U_{i}$ open. Thus $V_{1} \cap V_{1} \cdot t=V_{1} \cap V_{2} \subseteq U_{1} \cap U_{2}=\varnothing$. But this contradicts the assumption above, so $x_{0}$ is a rest point.

Corollary 5.9. If $(X ; S) \in \mathscr{I S}$ has the Conley index of a hyperbolic critical point and $\mathscr{A N}(X ; S)$ is non-empty, then $S$ contains a fixed point of the flow.

Proof. The Conley index of a hyperbolic critical point is $\Sigma^{n}$, the homotopy type of a pointed $n$-sphere. Thus $\chi(X ; S)=(-1)^{n} \neq 0$.

Corollary 5.10. Suppose $(X ; S) \in \mathscr{S}$ with $S$ homeomorphic to a circle and $f$ is a self-map on $(X ; S)$. If $\mathscr{A N}(X ; S)$ is non-empty and $L(f ; X, S) \neq 0$, then for every $g$ homotopic to fas flow maps, there exists a fixed point of the flow $x$ with $x \in S$ and $g(x)=x$.

Proof. As in theorem 5.7, it suffices to consider $g=f$. If $L(f ; X, S) \neq 0$, then Fix $\left(\left.f \circ \varphi_{i}\right|_{s}\right) \neq \varnothing$ for sufficiently large $t$. As $f$ is equivariant, if $f \circ \varphi_{t}(x)=x$, then $f \circ \varphi_{1} \circ \varphi_{s}(x)=\varphi_{s}(x)$ for every $s \in \mathbb{R}$. It follows that the limit sets $\omega(x)$ and $\omega^{*}(x)$ are also fixed by $f$. If $S$ does not consist of a single periodic orbit, then $\omega(x)$ and $\omega^{*}(x)$ are fixed points. If $S$ is periodic with period $\tau$, then for large $n, f=f \circ \varphi_{n \tau}=$ id. But then for large $t \neq n \tau, \mathrm{id} \neq \varphi_{t}=f \circ \varphi_{t}=\mathrm{id}$.

Remarks. (i) The requirement that $S$ be an attractor in $f^{-1}(S)$ is satisfied if $S$ is a union of components of $f^{-1}(S)$, in particular if $S=f^{-1}(S)$. It is also satisfied if $S$ is an attractor in $X$ with $S \subseteq f^{-1}(S)$.

(ii) Just as $\mathrm{CH}_{*}$ has the special case $C H_{*}(S ; S)=H_{*}(S)$ for $S$ a compact metric space, this theorem has as a special case the Lefschetz theorem for compact absolute neighbourhood retracts (with $T(f ; S, S)=0$ ). However, this theorem applies in two cases in which the classical theorem does not. First, $S$ can be an ANR with $\mathscr{A} \mathcal{N}(X ; S)$ non-empty and $L(f ; X, S) \neq L(f ; S, S)=0$ (cf. example 5.17). Second, $S$ can fail to be an ANR, but can embed in $X$ so that $\mathscr{A} \mathcal{N}(X ; S)$ is non-empty and $L(f ; X, S) \neq 0$.

(iii) The time estimate $T(f ; X, S)$ is not necessary for fixed points to exist. However, to prove their existence by generating self-maps on index pairs, the time is required, and is in general non-zero. For example, the flow $\dot{x}=x$ on $\mathbb{R}$ admits self-map $f(x)=x / 2 . S=\{0\}$ is an isolated invariant set, and if $(N, L)$ is an index pair for $S$ in $\mathbb{R}$, then there is a point $x_{0}>0$ with $x_{0} \in L$ and $\left[0, x_{0}\right) \subseteq N \backslash L$. In particular, $f\left(x_{0}\right)=x_{0} / 2 \in N \backslash L$ and is only carried into $L$ by flowing forward for time $\ln (2)$. Thus $T(f ; \mathbb{R}, S)=\ln (2)$, yet fixed points of $f \circ \varphi_{t}$ exist for all $t$. 
There is also a version of the theorem for pairs $(X, A ; S)$ in $\mathscr{I} \mathscr{Y}$. If $(X, A)$ and $(Y, B)$ are closed pairs of flows with $X \subseteq Y$ and $A=X \cap B$, define $f:\left(X, A ; f^{-1}(S)\right) \rightarrow(Y, B ; S)$ to be a self-map on $(X, A ; S)$ if it is a self-map on $(X ; S)$. For such a map we have the commutative diagram

$$
\begin{aligned}
& \cdots \rightarrow C H_{*}(A ; A \cap S) \rightarrow C H_{*}(X ; S) \rightarrow C H_{*}(X, A ; S) \rightarrow \cdots \\
& \left(\left.i\right|_{A}\right)_{*}^{-1} \circ\left(\left.f\right|_{A}\right)_{*} \downarrow \quad i_{*}^{i-1} \circ f_{*} \downarrow \quad \quad{ }_{*}^{i-1} \circ f_{*} \downarrow \\
& \cdots \rightarrow C H_{*}(A ; A \cap S) \rightarrow C H_{*}(X ; S) \rightarrow C H_{*}(X, A ; S) \rightarrow \cdots
\end{aligned}
$$

If $\mathrm{CH}_{*}(X ; S)$ and $\mathrm{CH}_{*}(A ; A \cap S)$ are finitely generated, then $\mathrm{CH}_{*}(X, A ; S)$ is finitely generated. We can then define a relative Conley-Lefschetz number $L(f ; X, A, S)$ with (from the exact sequence)

$$
L(f ; X, S)=L\left(\left.f\right|_{A} ; A, A \cap S\right)+L(f ; X, A, S) .
$$

The theorem then becomes:

THEOREM 5.11. If $f$ is a self-map on $(X, A ; S) \in \mathscr{I S}$ and $S$ admits an index pair $\left(N, N_{0}\right) \in \mathscr{A N}(X ; S)$ with $\left(N / N_{0},(N \cap A) /\left(N_{0} \cap A\right)\right)$ an $A N R$ pair, then $L(f ; X, A, S)$ is defined. Further, if $L(f ; X, A, S) \neq 0$, then for every $g$ homotopic to $f$ as flow maps and every $t \geq T(g ; X, S)$, the composition $g \circ \varphi_{3}$, has fixed points in $S \cap \mathrm{cl}_{X}(X \backslash A)$.

Proof. Construct $N^{T}, N_{0}^{T}, U^{T}$ as in the proof of theorem 5.7. Consider the maps

$$
\left(\frac{N^{T}}{N_{0}^{T}}, \frac{\left(U^{T} \cup\left(N^{T} \cap A\right)\right)}{N_{0}^{T}}\right) \stackrel{\varphi_{T}^{T}}{\longrightarrow}\left(\frac{\left(f^{-1}\left(N^{T}\right)\right.}{f^{-1}\left(N_{0}^{T}\right)}, \frac{f^{-1}\left(N^{T} \cap A\right)}{f^{-1}\left(N_{0}^{T} \cap A\right)}\right) \stackrel{\bar{f}}{\longrightarrow}\left(\frac{N^{T}}{N_{0}^{T}}, \frac{N^{T} \cap A}{N_{0}^{T} \cap A}\right) .
$$

By construction, $U^{T} / N_{0}^{T}$ is an ANR; $\left(N^{T} \cap A\right) /\left(N_{0}^{T} \cap A\right)=(N \cap A)^{T} /\left(N_{0} \cap A\right)^{T}$ is an ANR by lemma 5.4. As all of the retractions involved preserve $A,\left(U^{T} \cap\right.$ $\left.\left(N^{T} \cap A\right)\right) / N_{0}^{T}$ is an ANR. Thus $\left(U^{T} \cup\left(N^{T} \cap A\right)\right) / N_{0}^{T}$ is an ANR, with $\left(U^{T} \cup\right.$ $\left(N^{T} \cap A\right)$ ) retracting onto $N_{0}^{T} \cup\left(N^{T} \cap A\right)$. The rest of the argument is unchanged by the presence of $A$, and gives fixed points in $\operatorname{cl}_{X}\left(N^{T} \backslash\left(U^{T} \cup\left(N^{T} \cap A\right)\right)\right)$, hence in $\operatorname{cl}_{X}(S \backslash(S \cap A))=S \cap \operatorname{cl}_{X}(X \backslash A)$.

CoRollary 5.12. Suppose $f$ is a self-map on $(X ; S) \in \mathscr{I S}$ and $S$ admits an index pair $\left(N, N_{0}\right) \in \mathscr{A N}(X ; S)$ with $(N, S)$ an $A N R$ pair. If $L(f \mid S ; S, S \cap S) \neq L(f ; X, S)$, then for every $g$ homotopic to $f$ as flow maps and every $t \geq T(g ; X, S)$, the composition $g \circ \varphi_{3}$, has fixed points in $\partial_{X} S$.

Proof. If $(N, S)$ is an ANR pair and $N / N_{0}$ is an ANR, then $\left(N / N_{0}, S / \varnothing\right)=$ $\left(N / N_{0},(N \cap S) /\left(N_{0} \cap S\right)\right)$ is an ANR pair. If $L(f \mid S ; S, S) \neq L(f ; X, S)$, then $L(f ; X, S, S) \neq 0$. Then, taking $A=S$ in 5.11 , there are fixed points in $\operatorname{cl}_{X}(X \backslash S) \cap$ $S=\partial S$.

$L(f \mid S ; S, S)$ is the ordinary Lefschetz number of $f \mid S$. If $L(f \mid S ; S, S)=0 \neq$ $L(f ; X, S)$, then the corollary gives fixed points in $\partial_{X} S$, even though $L(f ; X, S) \neq 0$ by itself only detects fixed points in $S$; and $L(f \mid S ; S, S)=0$ by itself gives no information. 
COROLlary 5.13. If $(X ; S) \in \mathscr{S} \mathscr{S}$ so that $S$ admits an index pair $\left(N, N_{0}\right) \in \mathscr{A N}(X ; S)$ with $(N, S)$ an $A N R$ pair, and $\chi(X ; S) \neq \chi(S)$, then there exist fixed points of the flow in $\partial S$.

Finally, we consider how the theorem behaves under continuation.

LEMMA 5.14 (local continuation of the Lefschetz theorem). Let $Y \times \Lambda$ be a parametrized family offlows, $\omega:[0,1] \rightarrow \mathscr{P}(Y)$ a path. Let $K=\pi \circ \omega([0,1]),\left(S_{t}, \lambda_{t}\right)=\omega(t)$, $S_{K}=\omega([0,1])$. Suppose that

(i) $S_{K} \subseteq X \subseteq Y \times K$ so that $F: X \rightarrow Y \times K$ is a self-map on $\left(X ; S_{K}\right)$;

(ii) there exists an $\left(N_{K}, N_{0 K}\right) \in \mathscr{A} \mathcal{N}\left(X \times K ; S_{K}\right)$ so that $\left(N_{t}, N_{0 t}\right)=\left(N_{K}, N_{0 K}\right) \cap$ $X_{t} \in \mathscr{A} \mathcal{N}\left(X_{t} ; S_{t}\right)$ for every $t$;

(iii) $j\left(\lambda_{t}, K\right): I\left(S_{1}, \lambda_{t}\right) \rightarrow I\left(S_{K}\right)$ is a homotopy equivalence for every $t$.

Then $L\left(F_{K} ; X, S_{K}\right)=L\left(F_{t} ; X_{t} ; S_{t}\right)$ for every $t$ (where $\left.X_{t}=\left(Y \times\left\{\lambda_{t}\right\}\right) \cap X\right)$. Further, if $L\left(F_{K} ; X \times K, S_{K}\right) \neq 0$, then for every $s \geq T\left(F_{K} ; X \times K, S_{K}\right)$ and $t \in[0,1]$, the composition $F_{t} \circ \varphi_{t, 3 s}$ has fixed points in $\left(S_{t}, \lambda_{t}\right)$.

Proof. Each $F_{t}$ is a self-map on $\left(X_{t} ; S_{t}\right)$. As noted in theorem 2.10 , we have $I\left(F_{K}\right) \circ j\left(\lambda_{t}, K\right)=j\left(\lambda_{t}, K\right) \circ I\left(F_{t}\right)$. At the homology level, $F_{K^{*}}$ and $F_{t^{*}}$ are conjugate and so have the same trace. The lemma follows by applying theorem 5.7 to each $\left(X_{t} ; S_{t}\right)$.

THEOREM 5.15 (global continuation of the Lefschetz theorem). Let $Y \times \Lambda$ be $a$ parametrized family of flows, $\omega:[0,1] \rightarrow \mathscr{S}(Y)$ a path. Let $K=\pi \circ \omega([0,1]),\left(S_{t}, \lambda_{t}\right)=$ $\omega(t), S_{K}=\omega([0,1])$. Suppose that

(i) $S_{K} \subseteq X \subseteq Y \times K$ so that $F: X \rightarrow Y \times K$ is a self-map on $\left(X, S_{K}\right)$;

(ii) there exist $\left\{J_{j}\right\}$ covering $[0,1]$ so that for each $j$ there exists an $\left(N_{j}, N_{0 j}\right) \in$ $\mathscr{A} \mathcal{N}\left(X \times J_{j} ; S_{j}\right)$ such that $\left(N_{t}, N_{0 t}\right)=\left(N_{j}, N_{0 j}\right) \cap X_{t} \in \mathscr{A} \mathcal{N}\left(X_{t} ; S_{t}\right)$ for every $t \in J_{j}$.

Then $L\left(F_{0} ; X_{0}, S_{0}\right)=L\left(F_{1} ; X_{t}, S_{t}\right)$ for every $t \in[0,1]$. Further, if $L\left(F_{0} ; X_{0}, S_{0}\right) \neq 0$, then there exists a $T \geq 0$ so that for every $t \in[0,1], s \geq T$ the composition $F_{t} \circ \varphi_{1,3 s}$ has fixed points in $\left(S_{t}, \lambda_{t}\right)$.

Proof. From the continuation theorem, there exist $\left\{K_{i}\right\}$ covering $[0,1]$ so that (iii) of lemma 5.14 holds on $K_{i}$. Let $K_{i j}=K_{i} \cap J_{j}$. Then $\left\{K_{i j}\right\}$ give a cover of $[0,1]$ with lemma 5.14 holding on each $K_{i j} . L\left(F_{t} ; X_{t}, S_{t}\right)$ is constant on each $K_{i j}$, so on $[0,1]$. Take $T=\max \left\{T\left(F_{i j} ; X_{i j}, S_{i j}\right)\right\}$. The result follows by applying lemma 5.14 to the $K_{i j}$.

COROLllary 5.16. If $X \times \Lambda$ is a parametrized family of flows, $\left(S_{\lambda}, \lambda\right) \in \mathscr{S}(X)$ with $\chi\left(X_{\lambda}, S_{\lambda}\right) \neq 0$, then if $\left(S_{\mu}, \mu\right) \in \mathscr{S}(X)$ continues to $\left(S_{\lambda}, \lambda\right)$ by a path $\omega:[0,1] \rightarrow \mathscr{S}(X)$ satisfying the ANR hypothesis of theorem 5.15, there exists a fixed point of the $\mu$-flow in $S_{\mu}$.

Example 5.17. Consider the family of flows on $\mathbb{R}^{3}$ given by

$$
\left\{\begin{array}{l}
\dot{\rho}=f_{1}(\rho, \lambda) \cos ^{2}(\phi)+f_{2}(\rho, \lambda) \sin ^{2}(\phi), \quad 0 \leq \lambda \leq 1, \\
\dot{\theta}=0, \\
\dot{\phi}=2 \cos (\phi) \sin (\phi),
\end{array}\right.
$$


with $f_{1}(\rho, \lambda)=-\rho^{2}\left(\rho^{2}-\lambda^{2}\right)\left(\rho^{2}+\lambda^{2}\right)^{-3 / 2}$ and $f_{2}(\rho, \lambda)=\rho^{3}\left(\rho^{2}-\lambda^{2}\right)^{2}\left(\rho^{2}+\lambda^{2}\right)^{-3}$. For each $\lambda$ the set of bounded orbits is $S_{\lambda}=\{\rho \leq \lambda\}$, so $S_{\lambda}$ is isolated and all $S_{\lambda}$ are related by continuation. At $\lambda=0, S_{\lambda}$ is a hyperbolic critical point with Conley index $h\left(\mathbb{R}^{3} ; S_{0}\right)=\Sigma^{2}$. For $\lambda>0$ the origin is a repeller $A_{\lambda}^{*}$, with dual attractor $A_{\lambda}=\{\rho=0\}$. As the system is independent of $\theta$, the map $g(\rho, \theta, \phi)=(\rho,-\theta, \phi)$ is a flow map. Further, for all $\lambda$ it is a self-map on $\left(\mathbb{R}^{3} ; S_{\lambda}\right),\left(\mathbb{R}^{3} ; A_{\lambda}\right)$ and $\left(\mathbb{R}^{3} ; A_{\lambda}^{*}\right)$ with $T\left(g ; \mathbb{R}^{3}, S_{\lambda}\right)=T\left(g ; \mathbb{R}^{3} ; A_{\lambda}\right)=T\left(g ; \mathbb{R}^{3} ; A_{\lambda}^{*}\right)=0$.

At $\lambda=0$ the sets $N_{2}=\{\rho \leq 1\}, N_{0}=\{\rho=1, \pi / 4 \leq \phi \leq 3 \pi / 4\}$ form an ANR index pair with $g^{-1}\left(N_{2}\right)=N_{2}, g^{-1}\left(N_{0}\right)=N_{0}$. Thus $L\left(g ; \mathbb{R}^{3} ; S_{0}\right)=L\left(g \mid N_{2}\right)-L\left(g \mid N_{0}\right)=$ -1. Similarly, for $\lambda>0$ the sets $\tilde{N}_{2}=\{\rho \leq 2 \lambda\}, \tilde{N}_{1}=\{\lambda / 2 \leq \rho \leq 2 \lambda\}$,

$$
\tilde{N}_{0}=\left\{\rho=2 \lambda, \tan ^{2}(\phi) \geq-f_{1}(2 \lambda, \lambda) / f_{2}(2 \lambda, \lambda)\right\}
$$

form an ANR index triple for $\left(S_{\lambda} ; A_{\lambda}, A_{\lambda}^{*}\right)$ with $g^{-1}\left(\tilde{N}_{i}\right)=\tilde{N}_{i}$ and $L\left(g \mid \tilde{N}_{2}\right)=1$, $L\left(g \mid \tilde{N}_{1}\right)=0, L\left(g, \tilde{N}_{0}\right)=2$. Thus $L\left(g ; \mathbb{R}^{3}, S_{\lambda}\right)=-1, L\left(g ; \mathbb{R}^{3} ; A_{\lambda}^{*}\right)=1, L\left(g ; \mathbb{R}^{3}, A_{\lambda}\right)=$ -2. Note that $L\left(g ; \mathbb{R}^{3}, A_{\lambda}\right)+L\left(g ; \mathbb{R}^{3}, A_{\lambda}^{*}\right)=L\left(g ; \mathbb{R}^{3}, S_{\lambda}\right)$. In [9] conditions are developed for which an attractor-repeller pair (or more generally, a Morse decomposition) has $L(f ; X, A), L\left(f ; X, A^{*}\right)$ and $L(f ; X, S)$ all defined and $L(f ; X, S)=$ $L(f ; \boldsymbol{X}, \boldsymbol{A})+L\left(f ; \boldsymbol{X}, A^{*}\right)$.

Thus $L\left(g ; \mathbb{R}^{3}, A_{\lambda}\right)$ detects fixed points of $g$ in $A_{\lambda}$, while $L\left(f \mid A_{\lambda}\right)=0$ does not. Also each plane $P\left(\theta_{0}\right)=\left\{\theta=\theta_{0}, \theta_{0}+\pi\right\}, \quad h\left(P(\theta) ; A_{\lambda} \cap P(\theta)\right)=\Sigma^{1} \vee \Sigma^{1}$ and $\chi\left(F ; A_{\lambda} \cap P(\theta)\right)=-2$, while $\chi\left(A_{\lambda} \cap P(\theta)\right)=0$. That is, $\chi\left(P(\theta) ; A_{\lambda} \cap P(\theta)\right)$ detects fixed points in $A_{\lambda} \cap P(\theta)$, while $\chi\left(A_{\lambda} \cap P(\theta)\right)$ does not.

Acknowledgements. The author is indebted to Charles Conley for all of his help and inspiration. He would also like to thank Edward Fadell and Joel Robbin for their advice and encouragement in this work, which forms part of the author's Ph.D. thesis under the direction of Professor Fadell. The author was supported in part by NSF Grant DMS-8320099.

\section{REFERENCES}

[1] C. Conley. Isolated Invariant Sets and the Morse Index. CBMS Regional Conference Series in Mathematics 38. AMS, Providence, RI (1978).

[2] C. Conley \& E. Zehnder. Morse type index theory for flows and periodic solutions for Hamiltonian systems. Comm. Pure Appl. Math. XXXVII (1984), 207-253.

[3] A. Dold. Lectures on Algebraic Topology. Springer-Verlag, Berlin (1972).

[4] A. Floer \& E. Zehnder. The equivariant Conley index and bifurcations of periodic solutions of Hamiltonian systems. Ergod. Th. \& Dynam. Sys. 8* (1988), 87-97.

[5] R. Franzosa. Index filtrations and the homology index braid for partially ordered Morse decompositions. Trans. AMS 298 (1986), 193-213.

[6] R. Franzosa. The connection matrix theory for Morse decompositions. Trans. AMS. To be published

[7] H. Kurland. The Morse index of an isolated invariant set is a connected simple system. $J$. Differential Equations 42 (1981), 234-259.

[8] H. Kurland. Homotopy invariants of repeller-attractor pairs. I. The Puppe sequence of an R-A pair. J. Differential Equations 46 (1982), 1-31.

[9] C. McCord. Mappings and Morse decompositions in the Conley index theory. Preprint.

[10] C. McCord. The connection map for attractor-repeller pairs. Trans. AMS. To be published. 
[11] J. Robbin \& D. Salamon. Dynamical systems, shape theory and the Conley index. Ergod. Th. \& Dynam. Sys. 8* (1988), 375-393.

[12] D. Salamon. Connected simple systems and the Conley index of isolated invariant sets. Trans. AMS 291 (1985), 1-41.

[13] E. H. Spanier. Algebraic Topology. McGraw-Hill, New York (1966). 